\title{
Stabilization of Uncertain Linear Systems: An LFT Approach
}

\author{
Wei-Min Lu, Kemin Zhou, Member, IEEE, and John C. Doyle
}

\begin{abstract}
This paper develops machinery for control of uncertain linear systems described in terms of linear fractional transformations (LFT's) on transform variables and uncertainty blocks with primary focus on stabilization and controller parameterization. This machinery directly generalizes familiar statespace techniques. The notation of $\mathcal{Q}$-stability is defined as a natural type of robust stability, and output feedback stabilizability is characterized in terms of $\mathcal{Q}$-stabilizability and $\mathcal{Q}$ detectability which in turn are related to full information and full control problems. Computation is in terms of convex linear matrix inequalities (LMI's), the controllers have a separation structure, and the parameterization of all stabilizing controllers is characterized as an LFT on a stable, free parameter.
\end{abstract}

\section{INTRODUCTION}

$\mathbf{L}$ INEAR fractional transformations (LFT's) have come to play an important role in control system design [36], [12], [14], [28], [7], [31], [44]. In this paper, we develop machinery for linear fractional uncertain systems, represented as an LFT of a constant matrix on a block structure which includes the transform variables and uncertainty blocks. This LFT machinery is a direct generalization of the now standard state-space machinery for linear system analysis and synthesis. One of the advantages of the use of LFT representations is that it facilitates manipulation using state-space-like machinery, and computation involves constant matrix manipulation. The LFT provides a uniform framework for realization, analysis, and synthesis for uncertain systems, and even streamlines some of the standard linear systems results [4].

We focus on synthesis of stabilizing controllers for systems with LFT representations. The notion of $\mathcal{Q}$-stability is employed for such systems; it is a natural generalization of the conventional notions of stability for linear systems, robust stability for linear fractional uncertain systems, and robust performance of linear fractional uncertain systems. In particular, for a linear fractional uncertain system with norm-bounded linear time varying (LTV) or nonlinear uncertainty, $\mathcal{Q}$-stability is a necessary and sufficient test for robust stability and robust performance, and $\mathcal{Q}$-stability, $\mathcal{Q}$-stabilizability, $\mathcal{Q}$-detectability can be characterized using linear matrix inequalities (LMI's)

Manuscript received August 6, 1993; revised March 25, 1994 and June 28, 1995. Recommended by Associate Editor, A. Vicino. This work was supported in part by the NSF, AFOSR, and ONR.

W.-M. Lu is with the Department of Electrical Engineering, California Institute of Technology, Pasadena, CA 91125 USA.

K. Zhou is with the Department of Electrical and Computer Engineering, Louisiana State University, Baton Rouge, LA 70803 USA.

J. C. Doyle is with the Department of Electrical Engineering/Control and Dynamical Systems, California Institute of Technology, Pasadena, CA 91125 USA.

Publisher Item Identifier S0018-9286(96)00385-6. which result in computationally appealing convex optimization problems:

We first consider the stabilization problem in several special cases, i.e., the full information feedback, partial information feedback, and constant output feedback. The construction for the output feedback (OF) synthesis problem is then achieved via a separation argument and two special problems: full information (FI) and full control (FC). Our approach is similar to that in [13] and directly generalizes standard stabilization results and state-space methods, while streamlining much of the development. In particular, in the FI case; it is shown that the solvability is equivalent to $\mathcal{Q}$-stability and is characterized by the positive definite solutions of an LMI, and the controllers can be chosen as constant feedbacks. Dual results hold for the $\mathrm{FC}$ case, and $\mathcal{Q}$-stabilizability and $\mathcal{Q}$-detectability are necessary and sufficient for robust stabilization by output feedback.

The OF controllers are represented by LFT's on the same block structures as the plants, and all stabilizing controllers are parameterized as an LFT on a free, stable parameter. An appealing feature of this approach is that the necessity portion of the controller parameterization relies heavily on elegant LFT machinery [36], [19], [44] and avoids the need for coprime factorizations (see [43], [12], and [30]). In a further generalization it is noted that all that is required for the separation principle to hold for the LFT systems is that the stability is invariant under certain system transformations and cascade interconnections. The machinery used here also can be generalized to deal with controller parameterization for nonlinear systems [25].

There are several interpretations for such control schemes that are LFT's on the same block structure as the plant. In the multidimensional system case, the block structure represents transform variables; and the resulting controller then provides dynamic feedback [16], [20]; for an uncertain linear system with structured parameteric or dynamical perturbations, which can be viewed as a linear parameter varying or linear dynamic varying system [39], [41], [37], [5], [2], the resulting controllers, which are dynamical and depend on the perturbations, therefore may be thought of as gain scheduled or dynamically scheduled [42], [31]; the application implications of such control schemes are obvious when the uncertainties can be measured or identified on-line [42], [37].

The control problems of linear systems whose coefficients depend on unknown parameters is also addressed in the context of other algebraic structures [24], [23], [5], [25] and the parameter-dependent, or gain-scheduled, control solutions are 
characterized [23], [5], [25]. One of the major differences between the proposed LFT approach and others are that the arbitrary perturbations, including LTV and nonlinear uncertainties, can also be treated by the LFT approach, where the $\mathcal{Q}$-stability exactly captures this feature. In addition, the proposed LFT framework provides a systematic approach for gain-scheduled control design, where control solutions can be analytically constructed by solving the corresponding LMI's independent of the parameters (see [28], [33], [27], and [31]), and therefore the conventional ad hoc point-wise controller design and curve-fitting procedures (see [39]) are avoided; moreover, the type of scheduling that results from this LFT approach guarantees the global stability and global performances even when the uncertainty varies arbitrarily fast and thus avoids any potential hazards arising from conventional scheduling [41], [39]. On the other hand, one disadvantage of the proposed approach, which emphasizes $\mathcal{Q}$-stabilization, is that unless the uncertainties are arbitrary time-varying operators, the stability analysis on which the synthesis is based is potentially conservative [32]. This conservativeness may be most troublesome when the parameters are slowly timevarying, since neither the slow variation nor the parameteric nature of the uncertainty is exploited. Thus the methods in this paper should be viewed as a possible aid in conventional scheduling rather than a replacement.

This paper is based on the conference paper [28], where the main results were presented. Some relevant results in particular the LMI characterizations, were also obtained in [33] in the parallel context of robust performance synthesis for linear fractional uncertain (discrete-time) systems. Though robust stability and robust performance can be uniformly treated as $\mathcal{Q}$-stability, the essential difference between the results in this paper and those in [33] lies in their approaches taken for synthesis problems; the approach using separation arguments for the stability synthesis problem in this paper cannot be naively extended to the performance synthesis problem because of the block-dependent nature of the designed controllers. Many extensions and generalizations have been done since the appearance of these two conference papers [34], [27], [31], [21]. More recently, the LMI treatment has been extended to cope with robust performance synthesis for continuous-time systems [17], [2], [22]. A fairly complete review of LMI's and their roles in control theory is given in [8], and a software toolbox for the numerical solutions to LMI's is available [18].

This paper is presented in an axiomatic fashion in the order: Section II-A, Section III-A, Section IV, and Section V. The remaining structure is as follows: In the Appendix, some background material about LFT's, $\mu$, and matrix dilation are reviewed. In Section II, we first present a general framework for linear systems with LFT descriptions, $\mathcal{Q}$-stability is defined and characterized, and the robust stability and robust performances for linear fractional uncertain systems is examined to give some motivations for the stability notions; the implication of the stability notions in multidimensional systems is given in Section VII-B. In Section III, the properties of stabilizability and detectability for the LFT systems are examined, in particular, the $\mathcal{Q}$-stabilizability and $\mathcal{Q}$-detectability are characterized in terms of LMI's. Some simple cases for the stabilization of linear fractional uncertain systems, including full information feedback, partial information feedback, and constant output feedback, are examined. In Sections IV and V, the stabilization problem is considered, and an axiomatic approach is proposed; in Section IV, the stabilization problems are stated, and the relevant system structural properties are examined; Section $\mathrm{V}$ is devoted to the solutions of the general output-feedback problems, the special FI, DF, FC, and OE problems are first treated to develop machinery; the stabilizing controller and controller parameterization are constructed from the special problems via separation arguments.

The notations used in this paper is quite standard. $Z^{+}$ denotes the set of nonnegative integers. For a matrix $M \in$ $\boldsymbol{R}^{n \times m}\left(\boldsymbol{C}^{n \times m}\right), M^{T}$ denotes the transpose of $M$ and $M^{*}$ the conjugate transpose of $M ; \bar{\sigma}(M)$ denotes the largest singular value of $M$ and $\rho(M)$ the spectral radius of $M$. The block diagonal matrix is denoted with specified diagonal blocks as $\Delta:=\operatorname{Diag}\left[\Delta_{1}, \cdots, \Delta_{r}\right]$. The upper and lower LFT's of $M$ on $N$ are denoted as $\mathcal{F}_{u}(M, N)$ and $\mathcal{F}_{l}(M, N)$, respectively. The Redheffer star product of $M$ and $N$ is $\mathcal{S}(M, N)$.

\section{LINEAR FRACTIONAL UNCERTAIN SYSTEMS AND STABILITY}

A control implication for LFT is the feedback structure. In this section, an LFT framework is proposed for the description of unccrtain linear systems where the perturbations enter the systems in feedback fashions. We will consider the general linear systems with LFT descriptions, and their implications for linear fractional uncertain systems are revealed afterwards.

\section{A. Systems with LFT Descriptions}

Consider a class of systems each of which can be represented as an (upper) LFT on some block structure $\Delta$, i.e.,

$$
G=\mathcal{F}_{u}\left(\left[\begin{array}{ll}
A & B \\
C & D
\end{array}\right], \Delta\right)
$$

with $(A, B, C, D) \in \boldsymbol{R}^{n \times n} \times \boldsymbol{R}^{n \times p} \times \boldsymbol{R}^{q \times n} \times \boldsymbol{R}^{q \times p}$. We will refer to this class of linear systems as LFT systems, and (1) is its transfer function. The block structure $\Delta$ is defined as follows

$$
\Delta:=\operatorname{Diag}\left[\lambda I_{n_{0}}, \delta_{1} I_{r_{1}}, \cdots, \delta_{s} I_{r_{s}}, \Delta_{1} \cdots \Delta_{f}\right]
$$

which may include repeated full blocks and has dimension $n \times n$; the block structure may have several interpretations. We define a scaling matrix set with respect to the block structure $\Delta$ for the system as

$$
\begin{aligned}
\mathcal{D}:=\left\{D \in C^{n \times n} \text { is nonsingular : } D \Delta\right. \\
\left.=\Delta D \text { for all } \Delta \in C^{n \times n} \text { with structure (2) }\right\} .
\end{aligned}
$$

By analogy with standard terminology, we can also give the representation (1) a state-space interpretation. As in the conventional one-dimensional systems, (nonsingular) state variable transformations are useful in the analysis and synthesis of LFT systems. However, not all transformations are allowed in this setting; if we think of the system has "state" vector $x$, then the admissible state variable transformations $x \mapsto T x$ is therefore specified. 
Definition II.1 (Admissible Transformation): Consider the LFT system (1) with block structure $\Delta$ defined by (2) and the corresponding scaling matrix set $\mathcal{D}$ by (3). If $T \in \mathcal{D}$, then the following system transformation

$$
\mathcal{F}_{u}\left(\left[\begin{array}{ll}
A & B \\
C & D
\end{array}\right], \Delta\right) \mapsto \mathcal{F}_{u}\left(\left[\begin{array}{cc}
T A T^{-1} & T B \\
C T^{-1} & D
\end{array}\right], \Delta\right)
$$

is said to be admissible.

It is remarked that the transfer function after the transformation does not change. We will further show that many other properties of LFT systems are also invariant under admissible transformations.

With the block structure $\Delta$ defined in (2) and the corresponding scaling matrix set (3), the $\mu$ and $\mathcal{Q}$ values of the matrix $A$ are well defined. Next, we give some stability notions in terms of those values; their implications are given in the next subsection.

Definition II.2 ( $\mu$-Stability and $\mathcal{Q}$-Stability): Consider the LFT system (1) (or matrix $A$ ). It is $\mu$-stable (with respect to $\Delta$ ) if $\mu_{\Delta}(A)<1$. It is $\mathcal{Q}$-stable (with respect to $\Delta$ ) if $\mathcal{Q}_{\Delta}(A)<1$, i.e., there is a $D \in \mathcal{D}$ such that $\bar{\sigma}\left(D A D^{-1}\right)<1$.

$\mathcal{Q}$-stability is a sufficient test for $\mu$-stability, but $\mu$-stability does not imply $\mathcal{Q}$-stability, in general. These two stability notions are equivalent if and only if $\mu_{\Delta}(A)=\mathcal{Q}_{\Delta}(A)$. The implications of $\mathcal{Q}$-stability and $\mu$-stability are given in the next subsection. In the following, we only consider $\mathcal{Q}$-stability, the $\mu$-stability is discussed in [26]. It is noted the $\mathcal{Q}$-stability can be given the following Lyapunov characterization [14], [32].

Lemma II-3: System $A$ with block structure $\Delta$ is $\mathcal{Q}$-stable if and only if there exists a $P \in \mathcal{D}$ with $P=P^{*}>0$ such that

$$
A P A^{T}-P<0
$$

where the matrix set $\mathcal{D}$ is defined as in (3).

The following structural property of LFT systems follows immediately from the properties of $\mu$ and $\mathcal{Q}$.

Theorem II-4: The $\mu$-stability and $\mathcal{Q}$-stability of LFT systems are invariant under the admissible (system) transformations.

Theorem II-5: Lct $A_{1}$ and $A_{2}$ be two system matrices with respect to the block structures $\Delta_{1}$ and $\Delta_{2}$, respectively. Then:

1) If the system matrix $\left[\begin{array}{cc}A_{1} & A_{12} \\ A_{21} & A_{2}\end{array}\right]$ with any compatibly dimensional matrices $A_{12}$ and $A_{21}$ is $\mathcal{Q}$ (or $\mu$ )-stable with respect to the block structure $\Delta:=\operatorname{Diag}\left[\Delta_{1}, \Delta_{2}\right]$, where $\Delta_{1}$ and $\Delta_{2}$ are independent, then $A_{1}$ and $A_{2}$ are also $\mathcal{Q}$ (or $\mu$ )-stable with respect to structures $\Delta_{1}$ and $\Delta_{2}$, respectively.

2) The system matrix $\left[\begin{array}{cc}A_{1} & A_{12} \\ 0 & A_{2}\end{array}\right]$ with any compatibly dimensioned matrix $A_{12}$ is $\mathcal{Q}$ (or $\mu$ )-stable with respect to the block structure $\Delta:=\operatorname{Diag}\left[\Delta_{1}, \Delta_{2}\right]$ if and only if $A_{1}$ and $A_{2}$ are also $\mathcal{Q}(\mu)$-stable with respect to structures $\Delta_{1}$ and $\Delta_{2}$, respectively.

Note that statement 1) holds in the case where the structures $\Delta_{1}$ and $\Delta_{2}$ are independent, while 2) holds even when $\Delta_{1}$ and $\Delta_{2}$ depend on each other. 2) implies that a cascade system is $\mathcal{Q}$ (or $\mu$ )-stable if and only if each subsystem is $\mathcal{Q}$ (or $\mu$ )-stable. The definitions of $\mu$-stability and $\mathcal{Q}$-stability are generalizations of the notions of conventional stability (see

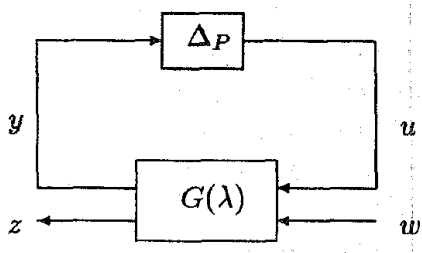

Fig. 1.

Section VII-B), robust stability, and robust performance [32], [25]. In the next section we will further reveal those facts for $\mathcal{Q}$-stability.

\section{B. Robust Stability of Linear Fractional Uncertain Systems}

A large class of uncertain linear systems can be described in terms of LFT's on some specified structures. Consider an uncertain system, where the uncertainty enters the systems in a feedback fashion as illustrated in Fig. 1, where $G$ is the nominal linear discrete-time system and $\Delta_{P}$ is the uncertainty which belongs to a designated uncertainty set. Both $G$ and $\Delta_{P}$ are causal, and the interconnection for the uncertain system is well-posed for each admissible uncertainty; $w$ is an input vector, and $z$ is an output vector. Thus, the transfer function of the uncertain system from $w$ to $z$ for each uncertainty $\Delta_{P}$ is represented in terms of the LFT formula as

$$
\mathcal{F}_{u}\left(G(\lambda), \Delta_{P}\right)
$$

In this paper, we assume that the uncertainty structure has the following form

$$
\Delta_{P}:=\operatorname{Diag}\left[\delta_{1} I_{r_{1}}, \cdots, \delta_{s} I_{r_{s}}, \Delta_{1}, \cdots, \Delta_{f}\right]
$$

where no blocks are repeated. Define a scaling matrix set corresponding to the uncertainty structure $\Delta_{P}$ as

$$
\begin{aligned}
\mathcal{D}_{P} & :=\left\{\operatorname{Diag}\left[D_{1}, \cdots, D_{s}, d_{1} I, \cdots, d_{f} I\right]\right. \\
& \left.\in C^{m \times m} \text { nonsingular: } D_{i} \in C^{r_{i} \times r_{i}}, d_{j} \in C\right\}
\end{aligned}
$$

where the dimensions of each block are the same as those of the corresponding block in $\Delta_{P}$.

Note that transfer function (from $\left[\begin{array}{l}u \\ w\end{array}\right]$ to $\left[\begin{array}{l}y \\ z\end{array}\right]$ ) of the nominal system $n_{0}$, can be described in terms of an LFT

$$
\begin{gathered}
G(\lambda)=\left[\begin{array}{ll}
G_{11}(\lambda) & G_{12}(\lambda) \\
G_{12}(\lambda) & G_{22}(\lambda)
\end{array}\right]:=\mathcal{F}_{u}\left(M, \lambda I_{n_{0}}\right) \\
M:=\left[\begin{array}{l|ll}
M_{11} & M_{12} & M_{13} \\
\hline M_{21} & M_{22} & M_{23} \\
M_{31} & M_{32} & M_{33}
\end{array}\right]
\end{gathered}
$$

where $\lambda$ is the transform variable, i.e., the delay operator. Therefore the transfer function in (5) is

$$
\begin{aligned}
\mathcal{F}_{u}\left(G(\lambda), \Delta_{P}\right) & =\mathcal{F}_{u}\left(\mathcal{F}_{u}\left(M, \lambda I_{n_{0}}\right), \Delta_{P}\right) \\
& =\mathcal{F}_{u}\left(M,\left[\begin{array}{cc}
\lambda I_{n_{0}} & 0 \\
0 & \Delta_{P}
\end{array}\right]\right)
\end{aligned}
$$

Thus the uncertain system is described as an LFT system

$$
\mathcal{F}_{u}(M, \Delta)=: \mathcal{F}_{u}\left(\left[\begin{array}{ll}
A & B \\
C & D
\end{array}\right], \Delta\right)
$$


where

$$
\begin{gathered}
A:=\left[\begin{array}{ll}
M_{11} & M_{12} \\
M_{21} & M_{22}
\end{array}\right] \quad B:=\left[\begin{array}{l}
M_{13} \\
M_{23}
\end{array}\right] \\
C:=\left[\begin{array}{lll}
M_{31} & M_{32}
\end{array}\right] \quad D:=M_{33}
\end{gathered}
$$

and the block structure with dimension $\left(n_{0}+m\right) \times\left(n_{0}+m\right)$

$$
\Delta:=\left[\begin{array}{cc}
\lambda I_{n_{0}} & 0 \\
0 & \Delta_{P}
\end{array}\right]
$$

Let $n:=n_{0}+m$; define a scaling matrix set corresponding to the block structure $\Delta$ as follows

$$
\begin{aligned}
\mathcal{D}:=\left\{\operatorname{Diag}\left[D_{1}, D_{2}\right] \in C^{n \times n}: D_{1} \in C^{n_{o} \times n_{o}}\right. \\
\text { is nonsingular, } \left.D_{2} \in \mathcal{D}_{P}\right\} .
\end{aligned}
$$

Suppose the nominal system is internally stable, i.e., $\rho\left(M_{11}\right)<1$. It is known that if the uncertainty $\Delta_{p}$ is linear time-invariant and bounded by 1 , then the notion of $\mu$ stability captures the robust stability and robust performances of the corresponding linear fractional uncertain systems [26] Next our emphasis is dealing with the uncertain structure $\Delta_{p}$ which includes time-varying uncertainty; we will consider the robust stability and performance of the linear fractional uncertain system given by (9) and establish the relations to the $\mathcal{Q}$-stability.

Let $l^{s \times t}$ denote the set of linear time-varying causal operators: $l_{2}^{t}\left(Z^{+}\right) \rightarrow l_{2}^{s}\left(Z^{+}\right)$. Consider the linear fractional uncertain system (9); the permissible uncertainty set is defined as

$$
\begin{array}{r}
B \Delta_{P}^{L T V}:=\left\{\Delta_{P}=\operatorname{Diag}\left[\delta_{1} I_{r_{1}}, \cdots, \delta_{s} I_{r_{s}}, \Delta_{1}, \cdots, \Delta_{f}\right]\right. \\
\left.\in l^{m \times m}:\left\|\Delta_{P}\right\|_{l_{2}} \leq 1\right\}
\end{array}
$$

where $\|\bullet\|_{l_{2}}$ is the $l_{2}$-induced norm of an LTV operator.

The transfer function of system $(9)$ is $\mathcal{F}_{u}\left(G(\lambda), \Delta_{P}\right.$ ) for each $\Delta_{P} \in B \Delta_{P}^{L T V}$. The system (9) is said to be robustly stable if the system is asymptotically stable for each fixed $\Delta_{P} \in B \Delta_{P}^{L T V}$. It has robust performance if the system is stable and has $l_{2}$-induced norm less than 1 for each $\Delta_{P} \in B \Delta_{P}^{L T V}$. The robust stability and robust performance problems have been extensively studied (see [29], [40], [35]). The analysis is reduced to the gain-analysis of some scaled systems. In fact, let $G(\lambda)$ be partitioned conformably with the block structure $\Delta_{P}$ as in (8). The following results are relatively new, but are now well known (cf. [40], [29], [35], and [9]).

Lemma II-6: Consider the uncertain system (8) with $\Delta_{P} \in$ $B \Delta_{P}^{L T V}$. Then:

1) It is robustly stable if and only if the nominal system is stable, i.e., $\rho\left(M_{11}\right)<1$, and there exists a positive definite matrix $D_{P} \in \mathcal{D}_{P}$ such that

$$
\sup _{|\lambda| \leq 1} \bar{\sigma}\left(D_{P} G_{11}(\lambda) D_{P}^{-1}\right)<1
$$

2) It has robust performance if and only if the nominal system is stable, and there exists a positive definite matrix $D_{Q} \in \mathcal{D}_{P}$ such that

$$
\sup _{|\lambda| \leq 1} \bar{\sigma}\left(\left[\begin{array}{cc}
D_{Q} & 0 \\
0 & I
\end{array}\right] G(\lambda)\left[\begin{array}{cc}
D_{Q}^{-1} & 0 \\
0 & I
\end{array}\right]\right)<1
$$

It is noted that the above results also hold for nonlinear uncertainty [9]. We further have the following LMI characterizations.

Theorem II-7: Consider the uncertain system (8) with $\Delta_{P} \in B \Delta_{P}^{L T V}$. Then:

1) It is robustly stable if and only if there exists a positive definite matrix $P \in \mathcal{D}$ such that

$$
A P A^{T}-P<0 \text {. }
$$

2) It has robust performance if and only if there exists a positive definite matrix $Q \in \mathcal{D}$ such that

$$
M\left[\begin{array}{cc}
Q & 0 \\
0 & I
\end{array}\right] M^{T}-\left[\begin{array}{cc}
Q & 0 \\
0 & I
\end{array}\right]<0 .
$$

It is known from the discussion in the previous section that the LMI conditions of robust stability and robust performance for a linear fractional uncertain system are exactly the conditions for $\mathcal{Q}$-stability for some LFT systems. Therefore, from Theorem II-7 and the definition of $\mathcal{Q}$-stability, we can conclude that the $\mathcal{Q}$-stability is an abstraction of robust stability and robust performance of a linear fractional uncertain system under LTV perturbations.

\section{STABILIZATION OF LFT SYSTEMS: STABILIZABILITY AND DETECTABILITY}

In this section, we will first examine the stabilizability and detectability of LFT systems in the context of $\mathcal{Q}$-stability, and then we will consider their robust control implications for linear fractional uncertain systems.

\section{A. Stabilizability and Detectability}

Consider an LFT system $y=G u$ with block structure $\Delta$ defined by (2)

$$
G=\mathcal{F}_{u}\left(\left[\begin{array}{ll}
A & B \\
C & D
\end{array}\right], \Delta\right)
$$

where $y$ is the measurement output and $u$ is control input. Suppose $(A, B, C, D) \in \boldsymbol{R}^{n \times n} \times \boldsymbol{R}^{n \times p} \times \boldsymbol{R}^{q \times n} \times \boldsymbol{R}^{q \times p}$, and assume further that $B$ and $C$ are of full column and row ranks, respectively, i.e., $\operatorname{Rank}(B)=p \leq n$ and $\operatorname{Rank}(C)=q \leq n$. The scaling matrix set $\mathcal{D}$ with respect to $\Delta$ is given by (3).

The notions of stabilizability and detectability play important roles in the stabilization problem; they are defined in terms of the following two special structures of (17), respectively

$$
G_{S F}=\mathcal{F}_{u}\left(\left[\begin{array}{cc}
A & B \\
I & 0
\end{array}\right], \Delta\right), \quad G_{O I}=\mathcal{F}_{u}\left(\left[\begin{array}{ll}
A & I \\
C & 0
\end{array}\right], \Delta\right)
$$

Definition III.2 (Stabilizability): System (17) is $\mathcal{Q}$ stabilizable if there exists a controller for the corresponding system $G_{S F}$

$$
K=\mathcal{F}_{u}\left(\left[\begin{array}{ll}
F_{11} & F_{12} \\
F_{21} & F_{22}
\end{array}\right], \Delta_{0}\right)
$$

such that the closed-loop system is $\mathcal{Q}$-stable with respect to the induced block structure. 
Definition III.3 (Detectability): System (17) is $\mathcal{Q}$ detectable if there exists a controller for the corresponding system $G_{O I}$

$$
K=\mathcal{F}_{u}\left(\left[\begin{array}{ll}
L_{11} & L_{12} \\
L_{21} & L_{22}
\end{array}\right], \Delta_{0}\right)
$$

such that the closed-loop system is $\mathcal{Q}$-stable with respect to the induced block structure.

It is known that for a one-dimensional system, the stabilizability is equivalent to the statement that the system can be stabilized by a constant state-feedback. Is this property still true for a general LFT system? The answer is positive for the $\mathcal{Q}$-case. But first, we shall see when the LFT system is $\mathcal{Q}$-stabilized by a constant state-feedback.

Suppose the LFT system is $\mathcal{Q}$-stabilized by a constant statefeedback matrix $F \in R^{p \times n}$, i.e., $\mathcal{Q}_{\Delta}(A+B F)<1$, then by the Lyapunov characterization of $\mathcal{Q}$-stability, there exists a $P \in \mathcal{D}$ with $P=P^{T}>0$ such that

$$
(A+B F) P(A+B F)^{T}-P<0 .
$$

If $\operatorname{Rank}(B)=p<n$ we can find a $B_{\perp} \in \boldsymbol{R}^{n \times(n-p)}$ such that $B^{T} B_{\perp}=0$ and $\operatorname{Rank}\left(B_{\perp}\right)=n-p$, then we have

$$
B_{\perp}^{T}(A+B F) P(A+B F)^{T} B_{\perp}-B_{\perp}^{T} P B_{\perp}<0
$$

i.e.,

$$
B_{\perp}^{T} A P A^{T} B_{\perp}-B_{\perp}^{T} P B_{\perp}<0 .
$$

So the solvability of the last LMI is necessary for the system to be constant-state-feedback $\mathcal{Q}$-stabilizable. Surprisingly, this condition is also sufficient as stated by the following proposition.

Proposition III-3: Consider the LFT system (17) with block structure $\Delta$, and $\operatorname{Rank}(B)=p<n$. Let $B_{\perp} \in \boldsymbol{R}^{n \times(n-p)}$ be such that $B^{T} B_{\perp}=0$ and $\left[\begin{array}{ll}B & B_{\perp}\end{array}\right]$ be invertible. There exists a constant state feedback $F$ such that $A+B F$ is $\mathcal{Q}$-stable with respect to the block structure $\Delta$ if and only if there exists a matrix $P \in \mathcal{D}$ with $P=P^{T}>0$ such that

$$
B_{\perp}^{T} A P A^{T} B_{\perp}-B_{\perp}^{T} P B_{\perp}<0 .
$$

Moreover, if $P \in \mathcal{D}$ with $P=P^{T}>0$ satisfies the above inequality, then a $\mathcal{Q}$-stabilizing constant state feedback matrix can be chosen as

$$
F=-\left(B^{T} P^{-1} B\right)^{-1} B^{T} P^{-1} A .
$$

Proof: By the definition of $\mathcal{Q}$-stability, there exists a constant feedback $F$ such that the closed-loop system matrix $A+B F$ is $\mathcal{Q}$-stable with respect to the block structure $\Delta$ if and only if there exists a $D \in \mathcal{D}$, such that

$$
\begin{aligned}
1 & >\inf _{F \in \boldsymbol{R}^{p \times n}} \bar{\sigma}\left(D(A+B F) D^{-1}\right) \\
& =\inf _{F \in \boldsymbol{R}^{p \times n}} \bar{\sigma}\left(D A D^{-1}+D B F D^{-1}\right) .
\end{aligned}
$$

Let

$$
V_{\perp}^{\mathcal{T}}=\left(B_{\perp}^{T}\left(D^{T} D\right)^{-1} B_{\perp}\right)^{-\frac{1}{2}} B_{\perp}^{T} D^{-1}
$$

then it is easy to check that $V_{\perp}^{T} V_{\perp}=I$ and $V_{\perp}^{T}(D B)=0$. By Lemma VII-8, we have

$$
1>\inf _{F \in \boldsymbol{R}^{p \times n}} \bar{\sigma}\left(D(A+B F) \cdot D^{-1}\right)=\bar{\sigma}\left(V_{\perp}^{T} D A D^{-1}\right)
$$

i.e.,

$$
\left(V_{\perp}^{T} D A D^{-1}\right)\left(V_{\perp}^{T} D A D^{-1}\right)^{T}<I .
$$

Take $P=\left(D^{T} D\right)^{-1}$, then $P \in \mathcal{D}$ and $P=P^{T}>0$; hence, we have

$$
\left(B_{\perp}^{T} P B_{\perp}\right)^{-\frac{1}{2}} B_{\perp}^{T} A P A^{T} B_{\perp}\left(B_{\perp}^{T} P B_{\perp}\right)^{-\frac{1}{2}}-I<0
$$

or

$$
B_{\perp}^{T} A P A^{T} B_{\perp}-B_{\perp}^{T} P B_{\perp}<0 .
$$

Moreover, if some $P \in \mathcal{D}$ with $P=P^{T}>0$ satisfies the above inequality, then we can construct a constant state feedback matrix $F$ via Lemma VII-8 such that $A+B F$ is $\mathcal{Q}$-stable.

In fact, let

$$
V_{0}^{T}=\left(B^{T}\left(D^{T} D\right) B\right)^{-1 / 2} B^{T} D^{T} .
$$

Then $\left[V_{0}, V_{\perp}\right]$ is unitary; thus $F D^{-1}=-\left(V_{0}^{T} D B\right)^{-1} V_{0}^{T}$ $D A D^{-1}$, or

$$
F=-\left(V_{0}^{T} D B\right)^{-1} V_{0}^{T} D A=-\left(B^{T} P^{-1} B\right)^{-1} B^{T} P^{-1} A .
$$

The following theorem gives another LMI characterization for $\mathcal{Q}$-stabilizability.

Theorem III-4: Consider the LFT system (17) with block structure $\Delta$. Then there exists a constant state feedback $F$ such that $A+B F$ is $\mathcal{Q}$-stable if and only if there exists a matrix $P \in \mathcal{D}$ with $P=P^{T}>0$ such that

$$
A P A^{T}-P-B B^{T}<0 \text {. }
$$

Moreover, if $P \in \mathcal{D}$ with $P=P^{T}>0$ satisfies the above inequality, then a $\mathcal{Q}$-stabilizing constant state feedback matrix can be chosen as

$$
F=-\left(B^{T} P^{-1} B\right)^{-1} B^{T} P^{-1} A .
$$

Proof: If $B$ is square and of full rank, then the result is straightforward. We thus consider the case where $\operatorname{Rank}(B)=p<n$. So we only need to show that both LMI characterizations (19) and (21) are equivalent.

The implication $(21) \Rightarrow(19)$ follows by observing that

$$
B_{\perp}^{T}\left(A P A^{T}-P-B B^{T}\right) B_{\perp}=B_{\perp}^{T}\left(A P A^{T}-P\right) B_{\perp} .
$$

To show the reverse implication, suppose there exists a positive definite matrix $Q \in \boldsymbol{F}^{n \times n}$. such that

$$
B_{\perp}^{T}\left(A Q A^{T}-Q\right) B_{\perp}<0 .
$$

Note that $\left(B_{\perp}^{T}\left(A Q A^{T}-Q\right) B\right)\left(B^{T} B\right)^{-2}\left(B^{T}\left(A Q A^{T}-P\right) B_{\perp}\right)$ is a symmetric matrix, then there exists $\alpha>0$ small enough such that

$B_{\perp}^{T}\left(A Q A^{T}-Q\right) B_{\perp}+\alpha\left(B_{\perp}^{T}\left(A Q A^{T}-Q\right) B\right)\left(B^{T} B\right)^{-2}$ $\cdot\left(B^{T}\left(A Q A^{\dot{T}}-Q\right) B_{\perp}\right)<0$. 
Let $P=\alpha Q$ which is positive definite, then the above inequality implies

$$
\left[\begin{array}{cc}
B_{\perp}^{T}\left(A P A^{T}-P\right) B_{\perp} & B_{\perp}^{T}\left(A P A^{T}-P\right) B \\
B^{T}\left(A P A^{T}-P\right) B_{\perp} & -\left(B^{T} B\right)^{2}
\end{array}\right]<0
$$

or

$$
\left[\begin{array}{l}
B_{\perp}^{T} \\
B^{T}
\end{array}\right]\left(A P A^{T}-P-B B^{T}\right)\left[B_{\perp} \quad B\right]<0
$$

which implies (17).

Using the above result we can easily get the following theorem.

Theorem $I I I-5$ : System $G$ is $\mathcal{Q}$-stabilizable if and only if there exists a constant feedback matrix $F$ such that $A+B F$ is $\mathcal{Q}$-stable with respect to the same block structure.

Proof: The sufficiency is obvious. As for the necessity, assume that the system can be $Q$-stabilized by a controller $K=\mathcal{F}_{u}\left(\left[\begin{array}{ll}F_{11} & F_{12} \\ F_{21} & F_{22}\end{array}\right], \Delta_{0}\right)$ where $\Delta_{0}$ depends on the system block structure $\Delta$. By the interconnection properties of LFT's, this statement is equivalent to the fact that the augmented system

$$
G_{a}=\mathcal{F}_{u}\left(\left[\begin{array}{cc|cc}
A & 0 & B & 0 \\
0 & 0 & 0 & I \\
\hline I & 0 & 0 & 0 \\
0 & I & 0 & 0
\end{array}\right],\left[\begin{array}{cc}
\Delta & 0 \\
0 & \Delta_{0}
\end{array}\right]\right)
$$

is $\mathcal{Q}$-stabilized by the constant feedback $\left[\begin{array}{ll}F_{22} & F_{21} \\ F_{12} & F_{11}\end{array}\right]$ with respect to the block structure $\Delta_{N}=\left[\begin{array}{cc}\Delta & 0 \\ 0 & \Delta_{0}\end{array}\right]$. Denote the scaling matrix set with respect to $\Delta_{N}$ by $\mathcal{D}_{N}$; then by the above proposition, there exists a positive definite matrix $P_{N}=:\left[\begin{array}{cc}P & P_{1} \\ P_{1}^{T} & P_{0}\end{array}\right] \in \mathcal{D}_{N}$ such that

$$
\left[\begin{array}{cc}
A & 0 \\
0 & 0
\end{array}\right] P_{N}\left[\begin{array}{cc}
A & 0 \\
0 & 0
\end{array}\right]^{T}-P_{N}-\left[\begin{array}{cc}
B B^{T} & 0 \\
0 & I
\end{array}\right]<0
$$

which implies

$$
A P A^{T}-P-B B^{T}<0 .
$$

Thus the above LMI has a solution $P>0$. The conclusion follows via the previous theorem if we can verify that $P \in \mathcal{D}$. Indeed, since $P_{N} \in \mathcal{D}_{N}, P_{N} \Delta_{N}=\Delta_{N} P_{N}$, i.e.,

$$
\left[\begin{array}{cc}
P & P_{1} \\
P_{1}^{T} & P_{0}
\end{array}\right]\left[\begin{array}{cc}
\Delta & 0 \\
0 & \Delta_{0}
\end{array}\right]=\left[\begin{array}{cc}
\Delta & 0 \\
0 & \Delta_{0}
\end{array}\right]\left[\begin{array}{cc}
P & P_{1} \\
P_{1}^{T} & P_{0}
\end{array}\right]
$$

for all complex matrices $\Delta$ and $\Delta_{0}$ with the given structures; in particular, (20) implies $P \Delta=\Delta P$. By the definition of the scaling matrix set $\mathcal{D}(3)$, we have $P \in \mathcal{D}$.

The dual notion $\mathcal{Q}$-detectability can be similarly characterized in terms of LMI's by some dual arguments. We have the following theorem.

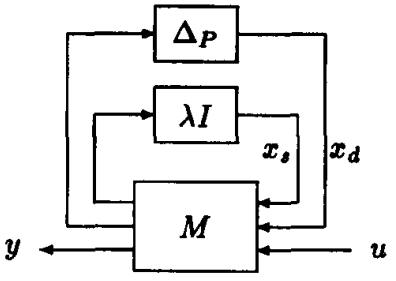

Fig. 2.

Theorem III-6: The given system $G(\Delta)$ is $\mathcal{Q}$-detectable if and only if there exists a matrix $P \in \mathcal{D}$ with $P=P^{T}>0$ such that

$$
A^{T} P A-P-C^{T} C<0
$$

In addition, there exists a constant output injection matrix $L$ such that $A+L C$ is $\mathcal{Q}$-stable with respect to the same frequency structure, and such an output injection matrix $L$ can be taken as

$$
L=-A P^{-1} C^{T}\left(C P^{-1} C^{T}\right)^{-1}
$$

for some $P \in \mathcal{D}$ with $P=P^{T}>0$ satisfying the LMI (21).

\section{B. Control of Linear Fractional Uncertain Systems: Constant Feedback}

In the last subsection, we considered the general notions of stabilizability for LFT systems. It was further shown that the stabilizability is reduced to constant full information feedback stabilization. In the following, we will further explore the implications of $\mathcal{Q}$-stabilizability and discuss several simple stabilization problems for the linear fractional uncertain systems. The system considered is represented as an LFT of a constant matrix on some block structure and is illustrated by the diagram in Fig. 2 . The uncertainty $\Delta_{P}$ is allowed to be structured and LTV, and varies in the uncertainty set $B \Delta_{P}^{L T V}$ as defined in (13). $x_{s}$ is the real state of the system and $x_{d}$ shows the signals from the uncertainty (and external disturbances if performance problem is considered). The transfer function is

$$
G=\mathcal{F}_{u}(M, \Delta)=: \mathcal{F}_{u}\left(\left[\begin{array}{ll}
A & B \\
C & D
\end{array}\right], \Delta\right)
$$

where $(A, B, C, D) \in \boldsymbol{R}^{n \times n} \times \boldsymbol{R}^{n \times p} \times \boldsymbol{R}^{q \times n} \times \boldsymbol{R}^{q \times p}, A$ is partitioned conformally with the block structure $\Delta:=$ $\operatorname{Diag}\left[\lambda I_{n 0}, \Delta_{P}\right]$ as $A=\left[A_{1} A_{2}\right]$, and $B$ and $C$ are of full column and row ranks, respectively; to avoid triviality, it is assumed that $\operatorname{Rank}(B)=p<n$ and $\operatorname{Rank}(C)=q<n$. The scaling matrix set $\mathcal{D}$ with respect to $\Delta$ is given by (3).

Constant Full Information Feedback: Suppose the full information $x:=\left[\begin{array}{l}x_{s} \\ x_{d}\end{array}\right]$ is available; the following statement directly follows from the discussions in the last subsection.

Theorem III-7: Consider the linear fractional uncertain system (26). There exists a constant full information feedback such that the system is robustly stable if and-only if there 
exists a positive definite solution in $P \in \mathcal{D}$ such that any of the following LMI's is satisfied

$$
\begin{aligned}
B_{\perp}^{T}\left(A P A^{T}-P\right) B_{\perp} & <0 \\
A P A^{T}-P-B B^{T} & <0
\end{aligned}
$$

and such a constant feedback is given by

$$
u=-\left(B^{T} P^{-1} B\right)^{-1} B^{T} A P^{-1} x .
$$

Therefore, the full information problem is solvable if and only if the related LFT system is $\mathcal{Q}$-stabilizable.

In the following, we will consider other two cases, i.e., only the real state of the system $x_{s}$ is available to the controller, and the measured output $y$ is available to the controller.

Constant State Feedback:

Theorem III-8: Consider system (22) where there exists a constant state feedback $u=K x_{s}$ such that the closedloop system is robustly stable if and only if there exists a positive definite matrix $P=\operatorname{Diag}\left[P_{1}, P_{2}\right] \in \mathcal{D}$ satisfying the following LMI's

$$
\begin{aligned}
B_{\perp}^{T}\left(A P A^{T}-P\right) B_{\perp} & <0, \\
A_{2} P_{2} A_{2}^{T}-P & <0 .
\end{aligned}
$$

Proof: By the definition of $\mathcal{Q}$-stabilizability, the closedloop system $A+B\left[\begin{array}{ll}K & 0\end{array}\right]$ is robustly stable iff there exists $D \in \mathcal{D}$ such that

$$
\inf _{K \in \boldsymbol{R}^{p \times n_{0}}} \bar{\sigma}\left(D\left(A+B\left[\begin{array}{ll}
K & 0
\end{array}\right]\right) D^{-1}\right)<1 .
$$

Let $D=\left[\begin{array}{cc}D_{1} & 0 \\ 0 & D_{2}\end{array}\right]$, then the above inequality is equivalent to

$$
\inf _{K \in \boldsymbol{R}^{p \times n_{0}}} \bar{\sigma}\left(D A D^{-1}+D B\left[K D_{1}^{-1} \quad 0\right]\right) .
$$

Let $V_{\perp}^{T}=\left(B_{\perp}^{T}\left(D^{T} D\right)^{-1} B_{\perp}\right)^{-1 / 2} B_{\perp}^{T} D^{-1}$ and $U_{\perp}^{T}=\left[\begin{array}{ll}0 & I\end{array}\right]$. It is easy to check that $V_{\perp}^{T} V_{\perp}=I$ and $V_{\perp}^{T}(D B)=0$, and $U_{\perp}^{T} U_{\perp}=I$ and $\left[K D_{1}^{-1} 0\right] U_{\perp}^{T}=0$. By Proposition VII-7, we have that (25) holds if and only if

$$
\max \left\{\bar{\sigma}\left(V_{\perp}^{T} D A D^{-1}\right), \bar{\sigma}\left(D A_{2} D_{2}^{-1}\right)\right\}<1 .
$$

Take

$P=\left(D^{T} D\right)^{-1}=\left[\begin{array}{cc}\left(D_{1}^{T} D_{1}\right)^{-1} & 0 \\ 0 & \left(D_{2}^{T} D_{2}\right)^{-1}\end{array}\right]=:\left[\begin{array}{cc}P_{1} & 0 \\ 0 & P_{2}\end{array}\right]$ then $P \in \mathcal{D}$ and $P=P^{T}>0$. Condition (26) can be rewritten as

$$
\bar{\sigma}\left(V_{\perp}^{T} D A D^{-1}\right)<1 \Leftrightarrow B_{\perp}^{T}\left(A P A^{T}-P\right) B_{\perp}<0
$$

and

$$
\bar{\sigma}\left(D A_{2} D_{2}^{-1}\right)<1 \Leftrightarrow A_{2} P_{2} A_{2}^{T}-P<0 .
$$

Thus, (25) holds iff we can find some matrix $P=P^{T}>0$ satisfying LMI's (23) and (24).

In the above theorem, such a state-feedback matrix $K$ is explicitly given in [21] by the use of Parrott's theorem (Lemma VII.6) and the condition (23) can be replaced by the following LMI

$$
A P A^{T}-P-B B^{T}<0
$$

which, as well as (23), implies $\mathcal{Q}$-stabilizability.

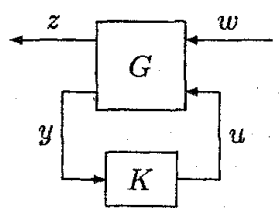

Fig. 3.

Constant Output Feedback: The following theorem gives a constant output feedback solution; it can be proven similarly to the above theorem by using Parrott's theorem (Proposition VII-7).

Theorem III-9: Consider system (9). Assume that $B_{\perp} \in$ $\boldsymbol{R}^{n \times(n-p)}$ is such that $B^{T} B_{\perp}=0$ and $\left[B . B_{\perp}\right]$ is invertible, and $C_{\perp} \in \boldsymbol{R}^{(n-q) \times n}$ is such that $C_{\perp} C^{T}=0$ and $\left[\begin{array}{c}C \\ C_{\perp}\end{array}\right]$ is invertible. Then there exists an admissible constant outputfeedback controller if and only if there exists a positive definite matrix $X \in \mathcal{D}$ such that the following two matrix inequalities hold

$$
\begin{array}{r}
B_{\perp}^{T} A X A^{T} B_{\perp}-B_{\perp}^{T} X B_{\perp}<0 \\
C_{\perp} A^{T} X^{-1} A C_{\perp}^{T}-C_{\perp} X^{-1} C_{\perp}^{T}<0 .
\end{array}
$$

Unfortunately, the matrix inequalities are not convex in $X$ and may not even have connected solution sets. Thus it is not clear that the search over $X$ is easier than the original problem of directly searching over controller gains.

It is a routine adaptation of Parrott's theorem to get a parameterization of all constant $\mathcal{Q}$-stabilizing output-feedback matrices. The above matrix inequality characterizations imply that the system is $\mathcal{Q}$-stabilizable and $\mathcal{Q}$-detectable. The above treatment in each case is suitable not only for the robust stabilization problem, but also for a robust performance synthesis problem by the standard trick of adding the additional performance block into $\Delta_{p}[15]$.

\section{CONTROL OF LFT SYSTEMS: PROBLEM STATEMENT AND SPECIAL STRUCTURES}

From now on, the stabilization problem for general LFT systems are investigated. The implication of the results for robust stabilization of linear fractional uncertain systems is obvious. In this section, we will examine the basic structures of the problems.

\section{A. Problem Statement}

Consider the control LFT system with standard block diagram in Fig. 3 where $G$ is the plant with two sets of inputs: the exogenous inputs $w$ and the control inputs $u$, and with two sets of outputs: the measured outputs $y$ and the outputs $z$ measuring the behavior of the system. The control problem is to design a feedback controller $K$ such that the resulting closed-loop system has some prescribed properties.

Suppose that the plant $G$ with block structure $\Delta$ is defined as

$$
G=\left[\begin{array}{ll}
G_{11} & G_{12} \\
G_{21} & G_{22}
\end{array}\right]=\mathcal{F}_{u}\left(\left[\begin{array}{c|cc}
A & B_{1} & B_{2} \\
\hline C_{1} & D_{11} & D_{12} \\
C_{2} & D_{21} & D_{22}
\end{array}\right], \Delta\right)
$$


where all matrices are real and have compatible dimensions with the related physical variables, the block structure is defined in (2), and the corresponding scaling matrix set is defined in (3). In addition, let the controller $K$ with block structure $\Delta_{0}$ be defined as

$$
K=\mathcal{F}_{u}\left(\left[\begin{array}{ll}
\hat{A} & \hat{B} \\
\hat{C} & \hat{D}
\end{array}\right], \Delta_{0}\right)
$$

In the following, it is assumed that the feedback system is well posed. The block structure $\Delta_{0}$ of the controller is determined by $\Delta$. In particular, for the output-feedback $\mathcal{Q}$ stabilization problem to be considered, the controller may have the same dependence on the block structure as the plant, i.e., $\Delta_{0}=\Delta$. This type of controllers for uncertain linear systems can be given a "gain scheduling" or "dynamic scheduling" interpretation; in the linear multidimensional system case, this means that dynamical feedback controllers are allowed (see Section VII-B).

Since we are only focusing on the $\mathcal{Q}$-stabilization problem, we will say that a feedback controller $K$ is admissible if it has the same dependence on the block structure as the plant and $\mathcal{Q}$-stabilizes (27), i.e., $\mathcal{F}_{l}(G, K)$ is $\mathcal{Q}$-stable with respect to the induced block structure $\operatorname{Diag}[\Delta, \Delta]$. Then the admissible controller set is denoted as $\mathcal{K}$. For convenience, this general synthesis problem is called the output feedback (OF) problem. The following two synthesis problems are considered in this paper:

- (Stabilization) Find a dynamical output feedback $K \in \mathcal{K}$ which $\mathcal{Q}$-stabilizes (27).

- (Parameterization) Characterize all controllers $K \in \mathcal{K}$ that $\mathcal{Q}$-stabilize (27), or more specifically, find an LFT system $J$ which has the same dependence on the block structure as the plant such that $\mathcal{K}=$ $\left\{\mathcal{F}_{l}(J, Q): Q\right.$ is a $\mathcal{Q}$-stable LFT system $\}$.

Note that $G$ is $\mathcal{Q}$-stabilized by $K$ if and only if $G_{22}$ is $\mathcal{Q}$-stabilized by $K$. Thus, the input $w$ and output $z$ do not affect the final stabilization results. They are reserved in the following treatment for technical reasons and to facilitate comparison with related problems where they are present. Next, we will examine some basic structural property for control LFT systems; we will use the following notation to represent an LFT system

$$
\left[\begin{array}{l|l}
A & B \\
\hline C & D
\end{array}\right]:=\mathcal{F}_{u}\left(\left[\begin{array}{ll}
A & B \\
C & D
\end{array}\right], \Delta\right)
$$

provided that the block structure is clear from context.

\section{B. Duality, Equivalence, and Special Problems}

Duality of Linear Systems: Duality plays an important role in the analysis and synthesis of linear systems. We now examine this notion further in the LFT setting employed in this paper. Algebraically, the dual system of an LFT system $G$ with block structure $\Delta$ is defined as the system represented by the transpose $G^{T}$ of $G$ with the same structure. It is

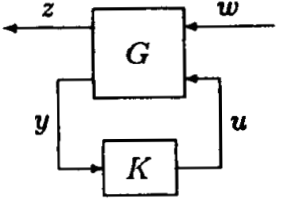

Fig. 4.

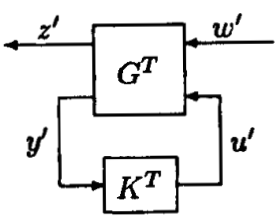

Fig. 5.

noted that if $G=\left[\begin{array}{l|l}A & A \\ \hline C & D\end{array}\right]$, then its dual system is $G^{T}=\left[\begin{array}{l|l}A^{T} & C^{T} \\ \hline B^{T} & \bar{D}^{T}\end{array}\right]$.

Next, we consider a feedback system with block diagram in Fig. 4 where the plant $G$ and the controller $K$ are assumed to be LFT's with respect to the same block structure $\Delta$. The dual structure of the above feedback system is shown in Fig. 5 whose plant and controller are the dual objects of $G$ and $K$, i.e., $G^{T}$ and $K^{T}$, respectively. It is routine to verify that $\mathcal{F}_{l}\left(G^{T}, K^{T}\right)=\left[\mathcal{F}_{l}(G, K)\right]^{T}$. In addition, $K \mathcal{Q}$-stabilizes $G$ with respect to the induced block structure $\Delta_{N}=\operatorname{Diag}[\Delta, \Delta]$ if and only if $K^{T} \mathcal{Q}$-stabilizes $G^{T}$ with respect to the block structure $\Delta_{N}$. Whence, as far as stabilization or other synthesis problems are concerned, we can obtain the results for system $G^{T}$ from those of its dual object $G$ if available.

Equivalence of LFT Systems: System equivalence notion will also play an important role in this paper. Two systems are said to be equivalent if every achievable closed-loop map of one system can be achieved by the other through some controller. More concretely, two LFT systems $G_{1}$ and $G_{2}$ with the same block structure are equivalent, if for all possible $K_{1}$, there exists an suitable $K_{2}$ depending on the same block structure as $K_{1}$, such that $\mathcal{F}_{l}\left(G_{1}, K_{1}\right)=\mathcal{F}_{l}\left(G_{2}, K_{2}\right)$; and also for all possible $K_{2}$, there is an suitable $K_{1}$ depending on the same block structure as $K_{2}$, such that $\mathcal{F}_{l}\left(G_{1}, K_{1}\right)=\mathcal{F}_{l}\left(G_{2}, K_{2}\right)$.

Special Structures: We consider four special structures which are related to the general OF problem whose associated plant $G$ with block structure $\Delta$ is given as in (27)

$$
G=\left[\begin{array}{c|cc}
A & B_{1} & B_{2} \\
\hline C_{1} & D_{11} & D_{12} \\
C_{2} & D_{21} & D_{22}
\end{array}\right] .
$$

- Full Information (FI) Problem: The corresponding plant has a structure as

$$
G_{F I}=\left[\begin{array}{c|cc}
A & B_{1} & B_{2} \\
\hline C_{1} & D_{11} & D_{12} \\
{\left[\begin{array}{l}
I \\
0
\end{array}\right]} & {\left[\begin{array}{l}
0 \\
I
\end{array}\right]} & {\left[\begin{array}{l}
0 \\
0
\end{array}\right]}
\end{array}\right]
$$


where the "state" and the disturbance are directly measured.

- Full Control (FC) Problem: The associated plant is given by

$$
G_{F C}=\left[\begin{array}{l|lll}
A & B_{1} & {[I} & 0
\end{array}\right]
$$

where two independent parts of the control input directly affect both the "state" and regulated output.

- Disturbance Feedforward (DF) Problem: The corresponding plant has a structure as

$$
G_{D F}=\left[\begin{array}{c|cc}
A & B_{1} & B_{2} \\
\hline C_{1} & D_{11} & D_{12} \\
C_{2} & I & 0
\end{array}\right]
$$

where the disturbance enters the measurement directly.

- Output Estimation (OE) Problem: The corresponding plant has the following structure

$$
G_{O E}=\left[\begin{array}{c|cc}
A & B_{1} & B_{2} \\
\hline C_{1} & D_{11} & I \\
C_{2} & D_{21} & 0
\end{array}\right]
$$

where the control input enter the regulated output directly.

Note that all of these special systems have the same block structures as $G$. The parameters in the special structures, however, do not necessarily refer to the same parameters of the above OF structure $G$. They are said to be special cases of the $\mathrm{OF}$ problem only in their structure. The reader is referred to [13] for motivations of different problems.

Structurally, FI and FC problems are dual and so are DF and $\mathrm{OE}$ problems. More precisely, $G_{F I}^{T}$ has an FC structure, $G_{F C}^{T}$ has an FI structure, and so on. In addition, FI and DF, and $\mathrm{FC}$ and $\mathrm{OE}$ are equivalent structures, respectively; this point will be made precise in the next section.

\section{STABILIZATION OF LFT SyStems}

In this section, we will show through the construction of solutions that the $\mathcal{Q}$-stabilizability and $\mathcal{Q}$-detectability are necessary and sufficient conditions for the output-feedback $\mathcal{Q}$-stabilization problem to be solvable.

\section{A. Solutions to Stabilization Problems}

In this subsection, we give the main results about the stabilization of LFT systems.

Theorem V-l (Stabilization): Given a system $G(27)$. There exists an admissible controller, i.e., $\mathcal{K} \neq \emptyset$, if and only if there exist two positive definite matrices $X \in \mathcal{D}$ and $Y \in \mathcal{D}$ such that the following two LMI's hold

$$
\begin{gathered}
A X A^{T}-X-B B^{T}<0 \\
A^{T} Y A-Y-C^{T} C<0 .
\end{gathered}
$$

Moreover, when the conditions hold, such a controller is given by

$$
K=\left[\begin{array}{c:c}
A+B_{2} F+L C_{2}+L D_{22} F & -L \\
\hline F & 0
\end{array}\right]
$$

with the same block structure $\Delta$ as the plant, where

$$
\begin{aligned}
& F=-\left(B_{2}^{T} X^{-1} B_{2}\right)^{-1} B_{2}^{T} X^{-1} A \\
& L=-A Y^{-1} C_{2}^{T}\left(C_{2} Y^{-1} C_{2}^{T}\right)^{-1} .
\end{aligned}
$$

The LMI conditions (34) and (35) in Theorem V-1 imply that the system is $\mathcal{Q}$-stable and $\mathcal{Q}$-detectable.

Theorem V-2 (Controller Parameterization): Assume that the conditions in Theorem V-1 are satisfied; then the admissible controller set for system (27) can be characterized by

$$
\mathcal{K}=\left\{\mathcal{F}_{l}(\mathcal{J}, Q): Q \text { is } \mathcal{Q} \text {-stable }\right\}
$$

where

$$
J=\left[\begin{array}{c|cc}
A+B_{2} F+L C_{2}+L D_{22} F & -L & B_{2}+L D_{22} \\
\hline F & 0 & I \\
-\left(C_{2}+D_{22} F\right) & I & -D_{22}
\end{array}\right]
$$

with same block structure $\Delta$ as the plant.

The next two subsections are mainly devoted to the constructive proofs of the above two main theorems. The necessity follows from Theorems III-9 and III-4; we will only consider the sufficiency in the following. We follow [13] to present a state-space-like approach to this problem without using any idea from coprime factorization, but using LFT machinery. We first reduce the OF problem into the simpler FI and OE problems, then solve the output feedback problem by a separation argument.

\section{B. Stabilization Problems for Special Systems}

In this subsection, we will develop machinery leading to a constructive proofs of the main theorems and consider the $\mathcal{Q}$-stabilization problems for special problems.

Admissible Controllers for FI and FC Systems: We " first examine the dual structures: FI and FC. Consider the plant $G_{F I}$ and (30) and $G_{F C}$ (31) with the same block structure $\Delta$; we immediately have the following statements using Theorem II-5.

Proposition V-3: Let $F$ be a constant matrix such that $A+B_{2} F$ is $\mathcal{Q}$-stable. Then a class of admissible controllers for the FI system can be parameterized as

$$
K_{F I}=\left[\begin{array}{ll}
F & Q
\end{array}\right]
$$

with $\mathcal{Q}$-stable $Q$.

Proposition V-4: Let $L$ be a constant matrix such that $A+L C_{2}$ is $\mathcal{Q}$-stable. Then a class of admissible controllers for $\mathrm{FC}$ can be parameterized as

$$
K_{F C}=\left[\begin{array}{l}
L \\
Q
\end{array}\right]
$$

with any $\mathcal{Q}$-stable $Q$. 
Equivalence Relations Between Special Problems: The equivalence relations between $\mathrm{DF}$ and FI, and $\mathrm{OE}$ and FC problems are examined in this subsection. We will construct the controller parameterizations for DF and $\mathrm{OE}$ problem through the equivalence relations. The different structures $G_{F I}, G_{F C}, G_{D F}$, and $G_{O E}$ are given as in the preceding section. We first have the following observation about DF and FI problems.

Proposition V-5: Consider FI and DF structures as given in Section V-A. Then

1) $G_{D F}=\left[\begin{array}{ccc}I & 0 & 0 \\ 0 & C_{2} & I\end{array}\right] G_{F I}$

2) $G_{F I}=\mathcal{S}\left(G_{D F}, P_{D F}\right)$, where $\mathcal{S}$ denotes the Redheffer star product and

$$
P_{D F}=\left[\begin{array}{c|cc}
A-B_{1} C_{2} & B_{1} & B_{2} \\
\hline 0 & 0 & I \\
{\left[\begin{array}{c}
I \\
-C_{2}
\end{array}\right]} & {\left[\begin{array}{l}
0 \\
I
\end{array}\right]} & {\left[\begin{array}{l}
0 \\
0
\end{array}\right]}
\end{array}\right] .
$$

Proof: 1) is easy; we only prove 2). Consider system $\mathcal{S}\left(G_{D F}, P_{D F}\right)$, let $x$ and $\hat{x}$ denote the "states" of $G_{D F}$ and $P_{D F}$, respectively; conduct a state transformation

$$
\left[\begin{array}{c}
x \\
\hat{x}
\end{array}\right] \mapsto\left[\begin{array}{c}
x-\hat{x} \\
\hat{x}
\end{array}\right]=\left[\begin{array}{cc}
I & -I \\
0 & I
\end{array}\right]\left[\begin{array}{l}
x \\
\hat{x}
\end{array}\right]
$$

which is admissible. The resulting interconnected system is as follows

$$
\left[\begin{array}{cc|cc}
A-B_{1} C_{2} & 0 & 0 & 0 \\
B_{1} C_{2} & A & B_{1} & B_{2} \\
\hline C_{1} & C_{1} & D_{11} & D_{12} \\
{\left[\begin{array}{c}
0 \\
C_{2}
\end{array}\right]} & {\left[\begin{array}{l}
I \\
0
\end{array}\right]} & {\left[\begin{array}{ll}
0 & I
\end{array}\right]} & {\left[\begin{array}{ll}
0 & 0
\end{array}\right]}
\end{array}\right]
$$

with respect to the block structure Diag $[\Delta, \Delta]$. The resulting transfer matrix is exactly $G_{F I}$, as claimed.

The following theorem follows the above observation immediately.

Theorem V-6:

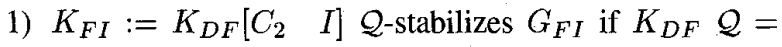
stabilizes $G_{D F}$. Furthermore

$$
\mathcal{F}_{l}\left(G_{F I}, K_{D F}\left[\begin{array}{ll}
C_{2} & I
\end{array}\right]\right)=\mathcal{F}_{l}\left(G_{D F}, K_{D F}\right) .
$$

2) Suppose that $A-B_{1} C_{2}$ is $\mathcal{Q}$-stable. Then $K_{D F}:=\mathcal{F}_{l}$ $\left(P_{D F}, K_{F I}\right) \mathcal{Q}$-stabilizes $G_{D F}$ if $K_{F I} \mathcal{Q}$-stabilizes $G_{F I}$. Furthermore, $\mathcal{F}_{l}\left(G_{D F}, \mathcal{F}_{l}\left(P_{D F}, K_{F I}\right)\right)$ $=\mathcal{F}_{l}\left(G_{F I}, K_{F I}\right)$.

Proof: 1) it is easy. As for 2), note that by Proposition $\mathrm{V}-5$, we have

$$
\begin{aligned}
\mathcal{F}_{l}\left(G_{F I}, K_{F I}\right) & =\mathcal{F}_{l}\left(\mathcal{S}\left(G_{F I}, P_{D F}\right), K_{F I}\right) \\
& =\mathcal{F}_{l}\left(G_{D F}, \mathcal{F}_{l}\left(P_{D F}, K_{F I}\right)\right) .
\end{aligned}
$$

The $\mathcal{Q}$-stability of the latter is confirmed by Theorem II-5-2), because of the structure of the closed-loop system in terms of the previous observation, the $\mathcal{Q}$-stability of $A-B_{1} C_{2}$, and the choice of $K_{F I}$.

This theorem shows that if $A-B_{1} C_{2}$ is $\mathcal{Q}$-stable, then problems FI and DF are equivalent.
Consider the DF system defined in the last section. Let $K_{D F}$ be an admissible controller. We next parameterize $K_{D F}$. By Theorem V-6, $K_{F I}=K_{D F}\left[C_{2} I\right] \mathcal{Q}$-stabilizes the corresponding $G_{F I}$. Then by Proposition V-3, given $F$ such that $A+B_{2} F$ is $\mathcal{Q}$-stable, there exists $Q$ which is $\mathcal{Q}$-stable such that $K_{F I}=\left[\begin{array}{ll}F & Q\end{array}\right] \mathcal{Q}$-stabilizes $G_{F I}$. Furthermore, $\mathcal{F}_{l}\left(J_{D F}, Q\right)=\mathcal{F}_{l}\left(P_{D F}, K_{F I}^{\prime}\right)$ where

$$
J_{D F}=\left[\begin{array}{c|cc}
A+B_{2} F-B_{1} C_{2} & B_{1} & B_{2} \\
\hline F & 0 & I \\
-C_{2} & I & 0
\end{array}\right] .
$$

Hence by Theorem V-6, $K_{D F}:=\mathcal{F}_{l}\left(J_{D F}, Q\right)$ stabilizes $G_{D F}$ for any $\mathcal{Q}$-stable $Q$.

Actually, the above constructed parameterization characterizes all admissible controllers (not just a class of them) for the DF system.

Proposition V-7: Consider the DF system. Suppose it is $\mathcal{Q}$ stabilizable and $A-B_{1} C_{2}$ is $\mathcal{Q}$-stable. Then all admissible controllers for the DF problem can be characterized by $K_{D F}=$ $\mathcal{F}_{l}\left(J_{D F}, Q_{0}\right)$ with $\mathcal{Q}$-stable $Q_{0}$, where $J_{D F}$ is given in (44).

Proof: From the above construction, it is known that the controllers expressed in the given LFT formula do $\mathcal{Q}$-stabilize $G_{D F}$. Let $K_{D F}$ be any admissible controller for $G_{D F}$, then $Q:=\mathcal{F}_{l}\left(\hat{J}_{D F}, K_{D F}\right)$ is $\mathcal{Q}$-stable where

$$
\hat{J}_{D F}=\left[\begin{array}{c|cc}
A & B_{1} & B_{2} \\
\hline-F & 0 & I \\
C_{2} & I & 0
\end{array}\right]
$$

since $\hat{J}_{D F}$ and $G_{D F}$ have the same parameters $\left(C_{2}, A, B_{2}\right)$ which are the only parameters affecting the stabilization results. We now claim that $K_{D F}=\mathcal{F}_{l}\left(J_{D F}, Q\right)$.

In fact, $\mathcal{F}_{l}\left(J_{D F}, Q\right)=\mathcal{F}_{l}\left(J_{D F}, \mathcal{F}_{l}\left(\hat{J}_{D F}, K_{D F}\right)\right)=$ : $\mathcal{F}_{l}\left(J_{t m p}, K_{D F}\right)$, where

$$
J_{t m p}=\left[\begin{array}{cc|cc}
A-B_{1} C_{2}+B_{2} F & -B_{2} F & B_{1} & B_{2} \\
-B_{1} C_{2} & A & B_{1} & B_{2} \\
\hline F & -F & 0 & I \\
-C_{2} & C_{2} & I & 0
\end{array}\right] .
$$

By conducting an admissible transformation, we have

$$
J_{t m p}=\left[\begin{array}{cc|cc}
A-B_{1} C_{2} & -B_{2} F & B_{1} & B_{2} \\
0 & A+B_{2} F & 0 & 0 \\
\hline 0 & -F & 0 & I \\
0 & C_{2} & I & 0
\end{array}\right]=\left[\begin{array}{ll}
0 & I \\
I & 0
\end{array}\right] .
$$

Hence, $\mathcal{F}_{l}\left(J_{D F}, Q\right)=\mathcal{F}_{l}\left(J_{t m p}, K_{D F}\right)=K_{D F}$. This shows that any admissible controller can be expressed in the form of $\mathcal{F}_{l}\left(J_{D F}, Q\right)$ for some $\mathcal{Q}$-stable $Q$.

Dually, we can also conclude that the structures FC and $\mathrm{OE}$ are equivalent provided $A-B_{2} C_{1}$ is $\mathcal{Q}$-stable. Furthermore, we have the following results about the structures $\mathrm{FC}$ and $\mathrm{OE}$.

Theorem V-8:

1) $K_{F C}:=\left[\begin{array}{c}B_{2} \\ I\end{array}\right] K_{O E} \quad \mathcal{Q}$-stabilizes $G_{F C}$ if $K_{O E} \mathcal{Q}-$ stabilizes $G_{O E}$. Furthermore

$$
\mathcal{F}_{l}\left(G_{F C},\left[\begin{array}{c}
B_{2} \\
I
\end{array}\right] K_{O E}\right)=\mathcal{F}_{l}\left(G_{O E}, K_{O E}\right) .
$$


2) Suppose that $A-B_{2} C_{1}$ is $\mathcal{Q}$-stable. Then $K_{O E}:=$ $\mathcal{F}_{l}\left(P_{O E}, K_{F C}\right) \mathcal{Q}$-stabilizes $G_{O E}$ if $K_{F C} \mathcal{Q}$-stabilizes $G_{F C}$. Furthermore, $\mathcal{F}_{l}\left(G_{O E}, \mathcal{F}_{l}\left(P_{O E}, K_{F C}\right)\right)=$ $\mathcal{F}_{l}\left(G_{F C}, K_{F C}\right)$.

Proposition V-9: Assume that $A-B_{2} C_{1}$ is $\mathcal{Q}$-stable. All admissible controllers for the OE problems can be characterized as $\mathcal{F}_{l}\left(J_{O E}, Q_{0}\right)$ with any $\mathcal{Q}$-stable $Q_{0}$, where $J_{O E}$ is defined as

$$
J_{O E}=\left[\begin{array}{c|cc}
A-B_{2} C_{1}+L C_{2} & L & -B_{2} \\
\hline C_{1} & 0 & I \\
C_{2} & I & 0
\end{array}\right]
$$

with $L$ such that $A+L C_{2}$ is $\mathcal{Q}$-stable.

\section{Stabilization by Output Feedback}

In this subsection, we will prove the main results based on the results provided in the last subsection. We only prove Theorem V-2; Theorem V-I is obtained by letting $Q=0$. The construction essentially involves reducing the OF problem to the simpler FI and FC problem. Consider system $G$ (27) with the block structure $\Delta$

$$
G=\left[\begin{array}{l|ll}
A & B_{1} & B_{2} \\
\hline C_{1} & D_{11} & D_{12} \\
C_{2} & D_{21} & D_{22}
\end{array}\right] .
$$

Controller Construction: We shall assume $D_{22}=0$ without loss of generality. In fact, if $D_{22} \neq 0$, then the mapping

$$
\hat{K}=K\left(I-D_{22} K\right)^{-1}=\mathcal{F}_{l}\left(\left[\begin{array}{cc}
0 & I \\
I & D_{22}
\end{array}\right], K\right)
$$

is well defined by the assumption that the closed-loop system is well posed. Define

$$
\hat{G}=\left[\begin{array}{c|cc}
A & B_{1} & B_{2} \\
\hline C_{1} & D_{11} & D_{12} \\
C_{2} & D_{21} & 0
\end{array}\right]
$$

then $\mathcal{F}_{l}(G, K)=\mathcal{F}_{l}(\hat{G}, \hat{K})$. Thus, if $\hat{K}$ is designed for the above structure, and $K$ can be obtained from Lemma VII-1 as

$$
K=\mathcal{F}_{u}\left(\left[\begin{array}{cc}
-D_{22} & I \\
I & 0
\end{array}\right], \hat{K}\right)=\mathcal{F}_{l}\left(\left[\begin{array}{cc}
0 & I \\
I & -D_{22}
\end{array}\right], \hat{K}\right) .
$$

This justifies the simplification.

Next, we construct the controllers for OF problem with $D_{22}=0$. Let $x$ denote the state of the system $G$. Since $\left(A, B_{2}\right)$ is $\mathcal{Q}$-stabilizable, there is a constant matrix $F$ such that $A+B_{2} F$ is $\mathcal{Q}$-stable. Note that $\left[\begin{array}{ll}F & 0\end{array}\right]$ is actually a special FI stabilizing controller. Let

$$
v=u-F x
$$

then the system can be broken into two subsystems $G_{1}$ and $G_{t m p}$ as shown pictorially in Fig. 6 with

$$
G_{1}=\left[\begin{array}{c|cc}
A+B_{2} F & B_{1} & B_{2} \\
\hline C_{1}+D_{12} F & D_{11} & D_{12}
\end{array}\right]
$$

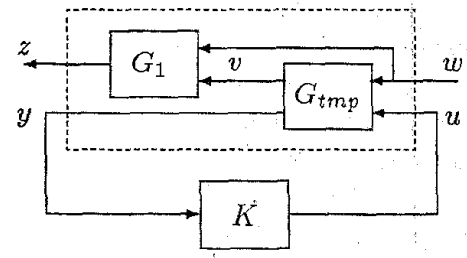

Fig. 6.

which is $\mathcal{Q}$-stable, and

$$
G_{t m p}=\left[\begin{array}{c|cc}
A & B_{1} & B_{2} \\
\hline-F & 0 & I \\
C_{2} & D_{21} & 0
\end{array}\right]
$$

Both have the same block structure $\Delta$ as the original plant. Since $G_{1}$ is $\mathcal{Q}$-stable, by Theorem II-5 $K \mathcal{Q}$-stabilizes $G$ if and only if $K \mathcal{Q}$-stabilizes $G_{t m p}$. Note that $G_{t m p}$ is of $\mathrm{OE}$ structure. Let $L$ be such that $A+L C_{2}$ is $\mathcal{Q}$-stable. Since $A+B_{2} F$ is $\mathcal{Q}$-stable by construction, by Theorem V-8 all controllers $\mathcal{Q}$-stabilizing $G_{t m p}$ are given by

$$
K=\mathcal{F}_{l}(J, Q)
$$

where

$$
J=\left[\begin{array}{c|cc}
A+B_{2} F+L C_{2} & -L & B_{2} \\
\hline F & 0 & I \\
-C_{2} & I & 0
\end{array}\right] .
$$

In particular, if $Q=0$, then

$$
K=\left[\begin{array}{c|c}
A+B_{2} F+L C_{2} & -L \\
\hline F & 0
\end{array}\right]
$$

When $D_{22}$ is arbitrary, the conclusions in the main theorems follow by the transformation (41).

Uniqueness of Parameterization: Let us further examine Theorem V-2. The theorem says that any admissible controller $K$ can be characterized as an LFT of a $\mathcal{Q}$-stable parameter matrix $Q$, i.e., $K=\mathcal{F}_{l}(J, Q)$. In fact, such a $Q$ can be uniquely determined by $K$. To state this precisely, recall from the inversion formulas for LFT's in Lemma VII- 1 that we can solve the equation $K=\mathcal{F}_{l}(J, Q)$ to uniquely give

$$
Q=\mathcal{F}_{u}\left(J^{-1}, K\right)=\mathcal{F}_{l}(\hat{J}, K)
$$

where a little algebra shows that

$$
J^{-1}=\left[\begin{array}{c|cc}
A & B_{2} & L \\
\hline C_{2} & D_{22} & I \\
-F & I & 0
\end{array}\right]
$$

and

$$
\begin{aligned}
\hat{J} & =\left[\begin{array}{ll}
0 & I \\
I & 0
\end{array}\right] J^{-1}\left[\begin{array}{ll}
0 & I \\
I & 0
\end{array}\right]=\left[\begin{array}{c|cc}
A & L & B_{2} \\
\hline-F & 0 & I \\
C_{2} & I & D_{22}
\end{array}\right] \\
& =:\left[\begin{array}{ll}
\hat{J}_{11} & \hat{J}_{12} \\
\hat{J}_{21} & \hat{J}_{22}
\end{array}\right] .
\end{aligned}
$$


Note that $Q$ is stable if and only if $K$ stabilizes $\hat{J}_{22}$. But $\hat{J}_{22}=G_{22}$, so $Q$ is stable if and only if $K$ stabilizes $G$, as desired. We summarize this observation as follows.

Theorem V-10: Any admissible controller $K$ can be characterized as an LFT of a $\mathcal{Q}$-stable parameter matrix $Q$, i.e., $K=\mathcal{F}_{l}(J, Q)$ with $Q$ realized by

$$
Q:=\mathcal{F}_{l}(\hat{J}, K)
$$

where

$$
\hat{J}=\left[\begin{array}{c|cc}
A & L & B_{2} \\
\hline-F & 0 & I \\
C_{2} & I & D_{22}
\end{array}\right]
$$

and the realization for $K$ is $\mathcal{Q}$-stabilizable and $\mathcal{Q}$-detectable. Moreover, this characterization is unique for a given pair $F$ and $L$ satisfying the requirements stated in Theorem V-1.

\section{A Separation Principle for LFT Systems}

Observer-Based Controller: The above construction was conducted by reducing the synthesis of the problem to the independent synthesis of FI and OE problems. This reduction is based on the separation property. And it also leads to a separation structure for the resulting closed loop system.

Let $\hat{x}$ be the "state variables" for the central controller which is represented as follows

$$
\Theta:\left\{\begin{array}{l}
\Delta^{-1} \hat{x}=A \hat{x}+B_{2} u-L\left(y-C_{2} \hat{x}\right) \\
u=F \hat{x}
\end{array}\right.
$$

which has an observer structure. The corresponding closedloop system, under the admissible transformation $T:=$ $\left[\begin{array}{cc}I & 0 \\ -I & I\end{array}\right]$, has the following transformation

$$
\begin{aligned}
{\left[\begin{array}{cc|c}
A & B_{2} F & B_{1} \\
-L C_{2} & A+B_{2} F+L C_{2} & -L D_{21} \\
\hline C_{1} & D_{12} F & D_{11}
\end{array}\right] } \\
\mapsto\left[\begin{array}{cc|c}
A+B_{2} F & B_{2} F & B_{1} \\
0 & A+L C_{2} & B_{1}-L D_{21} \\
\hline C_{1}-D_{12} F & D_{12} F & D_{11}
\end{array}\right]
\end{aligned}
$$

i.e., the transformed system is decoupled into two separated $\mathcal{Q}$ stable subsystems: the state-feedback system and the outputinjection system; hence, it is also $\mathcal{Q}$-stable with respect to the new block structure $\Delta_{N}=\left[\begin{array}{cc}\Delta & 0 \\ 0 & \Delta\end{array}\right]$ by Theorem II-5, so is the original closed-loop system as desired.

Structure of Parameterized Closed-Loop Systems: The central controller for the controller parameterization (with $Q=$ 0 ) is an observer-based controller, and the observer $\Theta$ is given by (42) where $\hat{x}$ is the estimate of the state $x$ of the original system. Therefore, a parameterized controller has some separation structure, and the closed-loop system is structured by the diagram, found in Fig. 7.

Next, consider the closed-loop map which is also parameterized as

$$
T_{z w}=\mathcal{F}_{l}(G, K)=\mathcal{F}_{l}\left(G, \mathcal{F}_{l}(J, Q)\right)=: \mathcal{F}_{l}(T, Q) .
$$

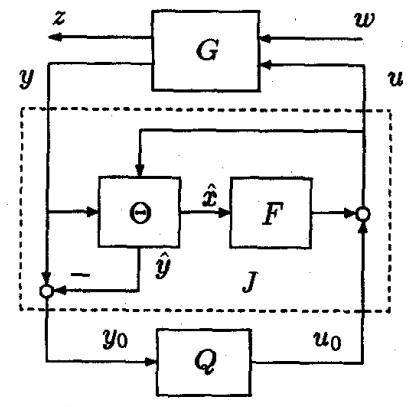

Fig. 7.

We have the following theorem about the structures of the closed-loop maps, whose proof is straightforward and is omitted.

Theorem $V$-11: Let the state feedback and output injection matrices $F$ and $L$ be chosen as in the previous theorem. Then the closed-loop maps are parameterized as $T_{z w}=\mathcal{F}_{l}(T, Q)$ with

$$
\begin{aligned}
T & =\left[\begin{array}{ll}
T_{11} & T_{12} \\
T_{21} & T_{22}
\end{array}\right] \\
& :=\left[\begin{array}{cc|cc}
A+B F & -B F & B_{1} & B \\
0 & A+L C & B_{1}+L D_{21} & 0 \\
\hline C_{1}+D_{12} F & -D_{12} F & D_{11} & D_{12} \\
0 & C & D_{21} & 0
\end{array}\right]
\end{aligned}
$$

and $Q$ is stable. Moreover, $T_{z w}$ is affine in $Q$, i.e.,

$$
T_{z w}=T_{11}+T_{12} Q T_{21}
$$

\section{CONCLUdING REMARKS}

We have developed machinery for analysis, stabilization, and controller parameterization for linear fractional uncertain systems. All of the manipulations have been conducted in the LFT framework and have based on some naturally defined stability notions for the LFT systems. A separation principle is confirmed for the linear fractional uncertain systems. It is noted that most of the results, including the separation theory, also hold in the $\mu$-stability case via simple change of notation. It is also remarked that although the stability notion captures the robust performances, the separation argument cannot be naively carried out for the performance synthesis problem. This is the essential difference between the approach used here and that in [33], [31].

It is observed that the separation property discussed in this paper holds in greater generality than for just the $\mathcal{Q}$ - and $\mu$-stability problems. All that is required for the separation proof is that the notion of stability satisfy two requirements: 1) stability invariance under a sufficiently rich set of similarity transformations, as in Theorem II-4, and -2) a certain structural property as given in Theorem II.5. It would clearly be possible to develop a more abstract axiomatic stabilization theory using these two properties. 


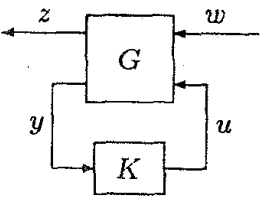

Fig. 8.

APPENDIX

\section{A. LFT's and $\mu$}

$L F T$ 's: The LFT formula arises naturally when we describe a well-posed feedback system as shown by the block diagram found in Fig. 8. The resulting input/output relation can be represented as $z=\mathcal{F}_{l}(G, K) w$, where $\mathcal{F}_{l}(G, K)$ is said to be the (lower) LFT of $G$ on $K$. More explicitly, suppose $G$ is partitioned conformably as

$$
G=\left[\begin{array}{ll}
G_{11} & G_{12} \\
G_{21} & G_{22}
\end{array}\right]
$$

Then

$$
\mathcal{F}_{l}(G, K):=G_{11}+G_{12} K\left(I-G_{22} K\right)^{-1} G_{21}
$$

provided the inverse is well defined [36]. Similarly, the (upper) LFT on $\Delta$, which corresponds to the feedback $\Delta$ around upper loop, is defined as

$$
\mathcal{F}_{u}(G, \Delta)=G_{22}+G_{21} \Delta\left(I-G_{11} \Delta\right)^{-1} G_{12} .
$$

The following observation is about the inversion property of an LFT [16].

Lemma VII-1: Suppose $G$ is partitioned as in (45). Let $P=\mathcal{F}_{l}(G, K)$. If $G, G_{12}$, and $G_{21}$ are square and invertible, and $\operatorname{det}\left(G_{11}-P\right) \neq 0$, then $K=\mathcal{F}_{u}\left(G^{-1}, P\right)$.

Redheffer Star Products: Consider the following wellposed feedback configuration shown in Fig. 9. Then the Redheffer star product of $Q$ and $M$ [36], denoted as $\mathcal{S}(Q, M)$, is the resulting transfer function, i.e., $\left[\begin{array}{l}y_{1} \\ y_{2}\end{array}\right]=\mathcal{S}(Q, M)\left[\begin{array}{l}u_{1} \\ u_{2}\end{array}\right]$. If $Q$ and $M$ are suitably partitioned as

$$
Q:=\left[\begin{array}{ll}
Q_{11} & Q_{12} \\
Q_{21} & Q_{22}
\end{array}\right], \quad M:=\left[\begin{array}{ll}
M_{11} & M_{12} \\
M_{21} & M_{22}
\end{array}\right]
$$

then $\left(I-Q_{22} M_{11}\right)^{-1}$ is well defined, and

$$
\begin{aligned}
& \mathcal{S}(Q, M) \\
& :=\left[\begin{array}{cc}
\mathcal{F}_{l}\left(Q, M_{11}\right) & Q_{12}\left(I-M_{11} Q_{22}\right)^{-1} M_{12} \\
M_{21}\left(I-Q_{22} M_{11}\right)^{-1} Q_{21} & \mathcal{F}_{u}\left(M, Q_{22}\right)
\end{array}\right] .
\end{aligned}
$$

Note that for any compatibly dimensioned matrix $K$, we have

$$
\mathcal{F}_{l}(\mathcal{S}(Q, M), K)=\mathcal{F}_{l}\left(Q, \mathcal{F}_{l}(M, K)\right)
$$

provided that the related LFT's are well defined.

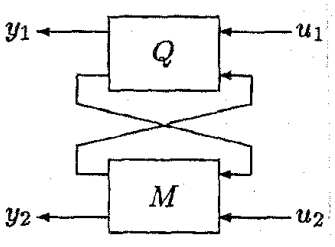

Fig. 9.

Structured Singular Values: Consider a matrix $M \in C^{n \times n}$ and an underlying block structure $\Delta$

$$
\Delta:=\operatorname{Diag}\left[\delta_{1} I_{r_{1}}, \cdots, \delta_{s} I_{r_{s}}, \Delta_{1}, \cdots, \Delta_{f}\right] .
$$

Corresponding to this block structure, define a set

$$
\begin{aligned}
\Delta:=\left\{\operatorname{Diag}\left[\delta_{1} I_{r_{1}}, \cdots, \delta_{s} I_{r_{s}}, \Delta_{1}, \cdots, \delta_{f}\right]:\right. & \\
& \left.\delta_{i} \in C, \Delta_{j} \in C^{m_{j} \times m_{j}}\right\} \subset C^{n \times n} .
\end{aligned}
$$

We first have the following definition structured singular value [9].

Definition VII.2: The structured singular value $\mu_{\Delta}(M)$ of a matrix $M$ with respect to structure $\Delta$ is defined as

$$
\mu_{\Delta}(M):=\sup _{\Delta \in \Delta}\left\{\frac{1}{\bar{\sigma}}: \operatorname{det}[I-\Delta M]=0\right\}
$$

unless no $\Delta \in \Delta$ makes $I-\Delta M$ singular, in which case $\mu_{\Delta}(M):=0$.

From the definition, it follows immediately that $\mu_{\Delta}(M)=$ $\rho(M)$ if $\Delta=\{\delta I: \delta \in C\}$ and $\mu_{\Delta}(M)=\bar{\sigma}(M)$ if $\Delta=$ $C^{n \times n}$.

The scaling matrix set $\mathcal{D}$ with respect to the block structure is defined as

$\mathcal{D}=\left\{D \in C^{n \times n}\right.$ nonsingular: $\left.D \Delta=\Delta D, \Delta \in \Delta\right\}$.

Definition VII.3: The $\mathcal{Q}$-value of $M$ with respect to structure $\Delta$ is defined as

$$
\mathcal{Q}_{\Delta}(M):=\inf _{D \in \mathcal{D}} \bar{\sigma}\left(D M D^{-1}\right)
$$

It is known that $\mathcal{Q}_{\Delta}(M)$ is an upper bound of $\mu_{\Delta}(M)$ [9], i.e., $\mu_{\triangle}(M) \leq \inf _{D \in \mathcal{D}} \bar{\sigma}\left(D M D^{-1}\right)$. The upper bound is achieved for the following special block structures:

1) $\Delta=\left\{\operatorname{Diag}\left[\delta I_{r}, \Delta\right]: \delta \in C, \Delta \in C^{(n-r) \times(n-r)}\right\}$.

2) $\Delta=\left\{\operatorname{Diag}\left[\Delta_{1}, \cdots, \Delta_{f}\right]: \Delta_{i} \in C^{m_{i} \times m_{i}}\right\} \in C^{n \times n}$, $f \leq 3$, where no blocks are repeated.

Remark VII.4: Both $\mu_{\Delta}(M)$ and $\mathcal{Q}_{\Delta}(M)$ are continuous functions of $M$ for fixed block structure $\Delta$.

To end this review, we state the following result known as main-loop theorem in [29].

Lemma VII-5: Given a matrix $M:-\left[\begin{array}{ll}M_{11} & M_{12} \\ M_{21} & M_{22}\end{array}\right]$ partitioned conformably with the block structure $\Delta=\operatorname{Diag}\left[\Delta_{1}, \Delta_{2}\right]$, then $\mu_{\Delta}(M)<1$ if and only if

$$
\mu_{\Delta_{2}}\left(M_{22}\right)<1, \quad \max _{\bar{\sigma}\left(\Delta_{2}\right) \leq 1} \mu_{\Delta_{1}}\left(\mathcal{F}_{l}\left(M, \Delta_{2}\right)\right)<1 .
$$




\section{B. Multidimensional Systems Represented by LFT's}

Consider a two-dimensional linear shift-invariant (LSI) system of order $\left(n_{1}, n_{2}\right)$ described by the Roesser state-space equation [35]

$$
\left\{\begin{array}{l}
x_{1}\left(k_{1}+1, k_{2}\right) \\
\quad=A_{11} x_{1}\left(k_{1}, k_{2}\right)+A_{12} x_{2}\left(k_{1}, k_{2}\right)+B_{1} u\left(k_{1}, k_{2}\right) \\
x_{2}\left(k_{1}, k_{2}+1\right) \\
\quad=A_{21} x_{1}\left(k_{1}, k_{2}\right)+A_{22} x_{2}\left(k_{1}, k_{2}\right)+B_{2} u\left(k_{1}, k_{2}\right) \\
y\left(k_{1}, k_{2}\right) \\
\quad=C_{1} x_{1}\left(k_{1}, k_{2}\right)+C_{2} x_{2}\left(k_{1}, k_{2}\right)+D u\left(k_{1}, k_{2}\right)
\end{array}\right.
$$

where $x_{1}\left(k_{1}, k_{2}\right) \in \boldsymbol{R}^{n_{1}}$ and $x_{2}\left(k_{1}, k_{2}\right) \in \boldsymbol{R}^{n_{2}}$ denote the state vectors, $u\left(k_{1}, k_{2}\right) \in \boldsymbol{R}^{p}$ the input vector, and $y\left(k_{1}, k_{2}\right) \in$ $\boldsymbol{R}^{q}$ the output vector. Define

$$
A:=\left[\begin{array}{ll}
A_{11} & A_{12} \\
A_{21} & A_{22}
\end{array}\right] \quad B:=\left[\begin{array}{l}
B_{1} \\
B_{2}
\end{array}\right] \quad C:=\left[\begin{array}{ll}
C_{1} & C_{2}
\end{array}\right]
$$

and $\Delta=\operatorname{Diag}\left[\lambda_{1} I_{n_{1}}, \lambda_{2} I_{n_{2}}\right]$, where $\lambda_{i} I$ can be interpreted as a backward shift operator. The transfer matrix for this system with zero initial conditions is

$$
G=D+C \Delta(I-\Delta A)^{-1} B=\mathcal{F}_{u}\left(\left[\begin{array}{ll}
A & B \\
C & D
\end{array}\right], \Delta\right)
$$

i.e., this system is represented as an LFT with respect to block structure $\Delta$. More generally, an $N$-dimensional discrete LSI system with order $\left(n_{1}, \cdots, n_{N}\right)$ can also be represented in terms of an LFT as (55) with respect to block structure

$$
\Delta=\operatorname{Diag}\left[\lambda_{1} I_{n_{1}}, \cdots, \lambda_{N} I_{n_{N}}\right]
$$

Define $\bar{U}^{N}:=\left\{\left(\lambda_{1}, \cdots, \lambda_{N}\right): \lambda_{i} \in C,\left|\lambda_{i}\right| \leq 1\right\}$. It is known that the $N$-dimensional system with system matrix $A$ defined above is internally stable if and only if $T\left(\lambda_{1}, \cdots, \lambda_{N}\right):=$ $\operatorname{Det}[I-\Delta A] \neq 0$ in $\bar{U}^{N}$ [5], [1]. Equivalently, $\mu_{\Delta}(A)<$ 1 ; in addition, the system is stable if there exists $P=$ $\operatorname{Diag}\left[P_{1}, \cdots, P_{N}\right]$ which is positive definite and $P_{i} \in \boldsymbol{R}^{n_{i} \times n_{i}}$, $i=1, \cdots, N$, such that the following Lyapunov inequality holds [1]

$$
A P A^{T}-P<0 \text {. }
$$

Note that the Lyapunov condition is equivalent to the $\mathcal{Q}$ stability.

\section{Parrott's Theorem} [12].

The following lemma is known as Parrott's Theorem [10],

Lemma VII-6: Suppose $(B, C, A) \in \boldsymbol{R}^{n_{1} \times m_{2}} \times \boldsymbol{R}^{n_{2} \times m_{1}} \times$ $\boldsymbol{R}^{n_{2} \times m_{2}}$; then

$$
\begin{aligned}
& \gamma_{0}:=\inf _{X \in \boldsymbol{R}^{n_{1} \times m_{1}}} \bar{\sigma}\left(\left[\begin{array}{cc}
X & B \\
C & A
\end{array}\right]\right) \\
& =\max \left\{\bar{\sigma}\left(\left[\begin{array}{ll}
C & A]
\end{array}\right], \bar{\sigma}\left(\left[\begin{array}{l}
B \\
A
\end{array}\right]\right)\right\}\right. \text {. }
\end{aligned}
$$

Let $\gamma>\gamma_{0}$, then those matrices $X \in R^{n_{1} \times m_{1}}$ such that $\bar{\sigma}\left(\left[\begin{array}{ll}X & B \\ C & A\end{array}\right]\right)<\gamma$ are exactly those of the form

$$
X=-Y A^{T} Z+\gamma\left(I-Y Y^{T}\right)^{1 / 2} W\left(I-Z^{T} Z\right)^{1 / 2}
$$

where $Y=B\left(\gamma^{2} I-A^{T} A\right)^{-1 / 2}, Z=\left(\gamma^{2} I-A A^{T}\right)^{-1 / 2} C$, and $W$ is an arbitrary contraction: $\bar{\sigma}(W)<1$.

As a corollary to the above lemma, the following result plays the key role in this paper.

Proposition VII-7: Consider the triple $(A, B, C) \in$ $\boldsymbol{R}^{n \times n} \times \boldsymbol{R}^{n \times p} \times \boldsymbol{R}^{q \times n}$ with $\operatorname{Rank}(B)=p<n$ and Rank $(C)=q<n$. Let $B_{\perp} \in \boldsymbol{R}^{n \times(n-p)}$ and $B_{0} \in \boldsymbol{R}^{p \times n}$ be such that $B_{\perp}^{T} B=0$ and $\left[\begin{array}{ll}B_{0} & B_{\perp}\end{array}\right]$ is unitary, and let $C_{\perp} \in$ $\boldsymbol{R}^{(n-q) \times n}$ and $C_{0} \in \boldsymbol{R}^{n \times q}$ be such that $C_{\perp} C^{T}=0$ and $\left[\begin{array}{c}C_{0} \\ C_{\perp}\end{array}\right]$ is unitary. Then

$$
\inf _{\boldsymbol{F} \in \boldsymbol{R}^{p \times q}} \bar{\sigma}(A+B F C)=\max \left\{\bar{\sigma}\left(B_{\perp}^{T} A\right), \bar{\sigma}\left(A C_{\perp}^{T}\right)\right\} .
$$

Proof: Notice that both $\left[\begin{array}{ll}B_{0} & B_{\perp}\end{array}\right]$ and $\left[\begin{array}{ll}C_{0} & C_{\perp}\end{array}\right]$ are unitary matrix. Then

$$
\begin{aligned}
\bar{\sigma}(A+B F C) & =\bar{\sigma}\left(\left[\begin{array}{ll}
B_{0} & B_{\perp}
\end{array}\right]^{T}(A+B F C)\left[\begin{array}{cc}
C_{0} & C_{\perp}
\end{array}\right]\right. \\
& =\bar{\sigma}\left(\left[\begin{array}{cc}
B_{0}^{T} A C_{0}^{T}+B_{0}^{T} B F C C_{0}^{T} & B_{0}^{T} A C^{T} \\
B_{\perp}^{T} A C_{0}^{T} & B_{\perp}^{T} A C_{\perp}^{T}
\end{array}\right]\right) \\
& =\max \left\{\bar{\sigma}\left(B_{\perp}^{T} A\right), \bar{\sigma}\left(A C_{\perp}^{T}\right)\right\}
\end{aligned}
$$

which follows the preceding lemma.

Given $\gamma>\max \left\{\bar{\sigma}\left(B_{\perp}^{T} A\right), \bar{\sigma}\left(A C_{\perp}^{T}\right)\right\}$, it is a routine adaptation of Parrott's theorem to get a parameterization of all matrices $F$ such that $\bar{\sigma}(A+B F C)<\gamma$. A special case of the above proposition is stated as follows.

Lemma VII-8: Assume $(A, B) \in \boldsymbol{R}^{n \times n} \times \boldsymbol{R}^{n \times p}$ and Rank $(B)=p<n$. Let $B_{\perp} \in \boldsymbol{R}^{n \times(n-p)}$ and $B_{0} \in \boldsymbol{R}^{p \times n}$ be such that $B_{\perp}^{T} B=0$ and $\left[B_{0} B_{\perp}\right]$ is unitary. Then

$$
\inf _{F \in \boldsymbol{R}^{p \times n}} \bar{\sigma}(A+B F)=\bar{\sigma}\left(B_{\perp}^{T} A\right)
$$

and the infimum is attained by $F=-\left(B_{0}^{T} B\right)^{-1} B_{0}^{T} A$.

Proof: Since $U:=\left[\begin{array}{ll}B_{0} & B_{\perp}\end{array}\right]$ is unitary

$$
\begin{aligned}
\inf _{F \in \boldsymbol{R}^{p \times n}} \bar{\sigma}(A+B F) & =\inf _{F \in \boldsymbol{R}^{p \times n}} \bar{\sigma}\left(U^{T}(A+B F)\right) \\
& =\inf _{F \in \boldsymbol{R}^{p \times n}} \bar{\sigma}\left(\left[\begin{array}{c}
B_{0}^{T} A+B_{0}^{T} B F \\
B_{\perp}^{T} A
\end{array}\right]\right) \\
& =\bar{\sigma}\left(B_{\perp}^{T} A\right) .
\end{aligned}
$$

Moreover the infimum is attained if $B_{0}^{T} A+B_{0}^{T} B F=0$ or $F=-\left(B_{0}^{T} B\right)^{-1} B_{0}^{T} A$.

The matrix $B_{0}$ in Lemma VII-8 can be chosen as $B_{0}=$ $B\left(B^{T} B\right)^{-1 / 2}$, in which case, $F=-\left(B^{T} B\right)^{-1} B^{T} A$.

\section{ACKNOWLEDGMENT}

The authors would like to thank Prof. A. Packard at the University of California at Berkeley for helpful discussion. They also gratefully acknowledge helpful comments on the previous versions of this paper from C. Beck, B. Bodenheimer, Y. Huang, J. Morris, F. Paganini, and J. Tierno, as well as the anonymous reviewers. 


\section{REFERENCES}

[1] B. D. O. Anderson, P. Agathoklis, E. I. Jury, and M. Mansour, "Stability and the matrix Lyapunov equation for discrete 2-dimensional systems," IEEE Trans. Circuits Syst., vol. CS-33, pp. 261-266, 1986.

[2] P. Apkarian and P. Gahinet, "A convex characterization of parameterdependent $\mathcal{H}_{\infty}$ controllers," IEEE Trans. Automat. Contr., vol. 40, pp. $853-864,1995$.

[3] C. Beck, "The computation issue of LMI's," in Proc. 1991 IEEE Conf. Decis. Contr., Brighton, England, 1991, pp. 1259-1260.

[4] C. L. Beck, W.-M. Lu, F. Paganini, R. D'Andrea, and J. C. Doyle, "A state-space theory for uncertain systems," IFAC 1996 World Congr., San Francisco, CA, July 1996.

[5] G. Becker and A. Packard, "Robust performance of linear parametrically varying systems using parametrically dependent linear feedback," Syst. Contr. Lett., vol. 23, pp. 205-215, 1994.

[6] N. K. Bose, Applied Multidimensional Systems Theory. New York: Van Nostrand Reinhold, 1982.

[7] S. P. Boyd and C. Barratt, Linear Controller Design: Limits of Performance. Englewood Cliffs, NJ: Prentice-Hall, 1991.

[8] S. P. Boyd, L. El Ghaoui, E. Feron, and V. Balakrishnan, Linear Matrix Inequalities in System and Control Theory. Philadelphia, PA: SIAM, 1994.

[9] M. A. Dahleh and I. J. Diaz-Bobillo, Control of Uncertain Systems: A Linear Programming Approach. . Englewood Cliffs, NJ: Prentice-Hall, 1994.

[10] C. Davis, W. M. Kahan, and H. F. Weinberger, "Norm-preserving dilations and their applications to optimal error bounds," SIAM J. Numer. Anal., vol. 19, pp. 445-469, 1982.

[11] J. C. Doyle, "Analysis of feedback systems with structured uncertainties," IEE Proc., Part D, vol. 133, pp. 45-56, 1982.

[12] __ Lecture notes in advances in multivariable control, ONR/Honeywell Wkshp., Mitineapolis, MN, 1984.

[13] J. C. Doyle, K. Glover, P. Khargonekar, and B. Francis, "State-space solutions to standard $\mathcal{H}_{2}$ and $\mathcal{H}_{\infty}$ control problems," IEEE Trans. Automat. Contr., vol. 34, pp. 831-847, 1989.

[14] J. C. Doyle, A. Packard, and K. Zhou, "Review of LFT's, LMI's and $\mu$," in Proc. 1991 IEEE Conf. Decis. Contr., Brighton, England, pp. $1227-1232$.

[15] J. C. Doyle, J. E. Wall, and G. Stein, "Performance and robustness analysis for structured uncertainty," in Proc. 21st IEEE CDC, pp. 629-636.

[16] R. Eising, "Realization and stabilization of 2-D systems," IEEE Trans. Automat. Contr., vol. AC-23, pp. 793-799, 1987.

[17] P. Gahinet and P. Apkarian, "A linear matrix inequality approach to $\mathcal{H}_{\infty}$ control," Int. J. Robust Nonlinear Contr., vol. 4, pp. 421-448, 1994.

[18] P. Gahinet, A. Nemirovskii, A. J. Laub, and M. Chilali, "The LMI control toolbox," in Proc. 1994 IEEE Conf. Decis. Contr., Orlando, FL, 1994, pp. 2038-2041.

[19] K. Glover, "A tutorial on Hankel-norm approximation," in From Date to Model, J. C. Willems, Ed. New York: Springer-Verlag, 1989.

[20] J. P. Guiver and N. K. Bose, "Causal and weakly causal 2-D filters with applications in stabilization," in Multidimensional Systems Theory, N. K. Bose, Ed. Reidel, 1985.

[21] Y. Huang and W.-M. Lu, "An LFT approach to artopilot design for missiles," in Proc. 1995 Am. Contr. Conf., Seattle, WA, June 1995.

[22] T. Iwasaki and R. E. Skelton, "A complete solution to the general $\mathcal{H}_{\infty}$ control problem: LMI existence conditions and state space formulas," in Proc. 1993, Am. Contr. Conf., San Francisco, CA, pp. 605-609.

[23] E. W. Kamen and P. P. Khargonekar, "On the control of linear systems whose coefficients are functions of parameters," IEEE Trans. Automat. Contr., vol. AC-29, pp. 25-33, 1984.

[24] P. P. Khargonekar and E. D. Sontag, "On the relation between stable matrix factorizations and regulable realizations of linear systems over rings," IEEE Trans. Automat. Contr., vol. AC-27, pp. 627-638, 1982.

[25] W.-M. Lu, "A state-space approach to parameterization of stabilizing controllers for nonlinear systems," IEEE Trans. Automat. Contr., vol. 40, no. 9, pp. 1576-1588, 1995 .

[26] _ _ "Control of uncertain systems: State-space characterizations, Ph.D. dissertation, California Inst. Technology, Pasadena, 1995.

[27] W.M. Lu and J. C. Doyle, "H ${ }_{\infty}$-control of LFT systems: An LMI approach," in Proc. 1992 IEEE Conf. Decis. Contr., Tucson, AZ, pp. 1997-2001.

[28] W.M. Lu, K. Zhou, and J. C. Doyle, "Stabilization of LFT systems," in Proc. 1991 IEEE Conf. Decis. Contr., Brighton, England, 1991, pp. $1239-1244$.

[29] A. Megretski, "Necessary and sufficient conditions of stability: A multiloop generalization of circle criterion," IEEE Trans. Automat. Contr., vol. 38, pp. 753-756, 1993.
[30] C. N. Nett, C. A. Jacobson, and N. J. Balas, "A connection between state-space and doubly coprime fractional representations," IEEE Trans. Automat. Contr., vol. AC-29, pp. 831-832, 1984.

[31] A. Packard, "Gain-scheduling via linear fractional transformations;" Syst. Contr. Lett., vol. 22, pp. 79-92, 1994.

[32] A. Packard and J. C. Doyle, "The complex structured singular value," Automatica, vol. 29, pp. 71-109, 1993.

[33] A. Packard, K. Zhou, P. Pandey, and G. Becker, "A collection of robust control problems leading to LMI's," in Proc. 1991 IEEE Conf. Decis. Contr., Brighton, England, 1991, pp. 1245-1250.

[34] A. Packard, K. Zhou, P. Pandey, J. Leonhardson, and G. Balas, "Optimal, constant I/O similarity scaling for full-information and statefeedback problems," Syst. Contr. Lett., vol. 19, pp. 271-280, 1992.

[35] F. Paganini and J. C. Doyle, "Analysis of implicitly defined systems," in Proc. 1994 IEEE Conf. Decis. Contr., Orlando, FL, pp. 3673-3678.

[36] R. M. Redheffer, "On a certain linear fractional transformation," J. Math. Phys., vol. 39, pp. 269-286, 1960.

[37] R. T. Reichert, "Dynamic scheduling of modern-robust-contrel autopilot designs for missiles," IEEE Contr. Syst. Mag., vol. 12, no. 5, pp. 35-42, 1992.

[38] R. P. Roesser, "A discrete state-space model for linear image processing," IEEE Trans. Automat. Contr., vol. AC-20, pp. 1-10, 1975.

[39] W. J. Rugh, "Analytical framework for gain scheduling," IEEE Contr. Syst. Mag., vol. 11, no. 1, pp. 79-84, 1991.

[40] J. S. Shamma, "Robust stability with time-varying structured uncertainty," IEEE Trans. Automat. Contr., vol. 39, pp. 714-724, 1994.

[41] J. S. Shamma and M. Athans, "Gain scheduling: Potential hazards and possible remedies," IEEE Contr. Syst. Mag., vol. 12, no. 3, pp. 101-107, 1992.

[42] G. Stein, "Adaptive flight control: A pragmatic view," in Applications of Adaptive Control, K. S. Narendra and R. V. Monopoli, Eds. New York: Academic, 1980

[43] D. C. Youla, H. A. Jabr, and J. J. Bongiono, "Modern Wiener-Hopf design of optimal controllets: The multivariable case," IEEE Trans. Automat. Contr., vol. AC-21, pp. 319-338, 1976.

[44] K. Zhou, J. C. Doyle, and K. Glover, Robust and Optimal Control. Englewood Cliffs, NJ: Prentice-Hall, 1995.

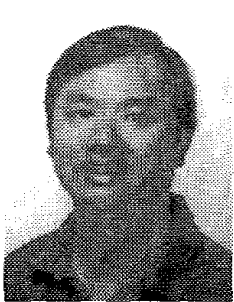

Wei-Min Lu was born in Changde, Hunan, China. $\mathrm{He}$ received the B.Sc. and M.Sc. degrees in automatic control from Tsinghua University, Beijing, China, in 1986 and 1989, respectively, and the Ph.D. degree in electrical engineering from the California Institute of Technology, Pasadena, CA, in 1995.

$\mathrm{He}$ is currently with the Department of Electrical. Engineering, California Institute of Technology and the Berkeley Center for Control and Identification, University of California, Berkeley. His research interests include modeling and control of uncertain systems, analysis and control of nonlinear systems, and discrete-event systems.

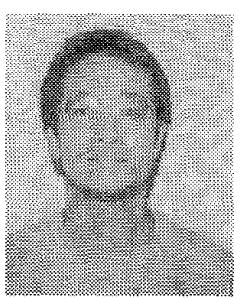

Kemin Zhou (S'86-M'88) was born in Wubu, China, on May 7, 1962. He received the B.S. degree in automatic control from Beijing University of Aeronautics and Astronautics, Beijing, in 1982, and the M.S.E.E. and $\mathrm{Ph} . \mathrm{D}$. degrees from the University of Minnesota, Minneapolis, in 1986 and 1988, re-spectively.

From 1982-1984, he was a Research Associate with Beijing University of Aeronautics and Astronautics. From 1988-1990, he was' a Research Fellow and Lecturer at the Department of Electrical. Engineering, California Institute of Technology, Pasadena, CA. Since 1990, he has been with the Department of Electrical and Computer Engineering, Louisiana State University, Baton Rouge. He is the principal author of the book Robust and Optimal Control. His current research interests include robust control, $\mathcal{H}_{2}$ and $\mathcal{H}_{\infty}$ optimal control, model/controller approximation, and industrial applications of control theory. 


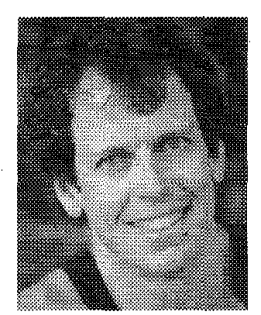

John C. Doyle received the B.S. and M.S. degrees in electrical engineering from the Massachusetts Institute of Technology, Cambridge, in 1977 and the $\mathrm{Ph} . \mathrm{D}$. degree in mathematics from the University of California, Berkeley, in 1984.

$\mathrm{He}$ is a Professor of Control and Dynamical Systems and Electrical Engineering at California Institute of Technology, Pasadena, and has been a Consultant to Honeywell Systems and Research Center since 1976. His theoretical research interests include modeling, identification, analysis and control of uncertain and nonlinear systems. His theoretical work has been applied throughout the aerospace industry and is gaining acceptance in the process control industry.

Dr. Doyle is the recipient of the Hickernell Award, the Eckman Award, the IEEE Control Systems Society Centennial Outstanding Young Engineer Award, and the Bernard Friedman Award. He is an NSF Presidential Young Investigator, an ONR Young Investigator, and has coauthored papers that have won the IEEE Baker Prize, the AACC Hugo Schuck Award, and two IEEE TRANSACTIONS George S. Axelby Outstanding Paper Awards. 